\title{
taxize: taxonomic search and retrieval in $\mathrm{R}$ [version 1; peer review: 3 approved]
}

\section{Scott A. Chamberlain 1 , Eduard Szöcs ${ }^{2}$}

${ }^{1}$ Biology, Simon Fraser University, Burnaby, Canada

${ }^{2}$ Institute for Environmental Sciences, University Koblenz-Landau, Landau, Germany

V1 First published: 18 Sep 2013, 2:191

https://doi.org/10.12688/f1000research.2-191.v1

Latest published: 28 Oct 2013, 2:191

https://doi.org/10.12688/f1000research.2-191.v2

\section{Abstract}

All species are hierarchically related to one another, and we use taxonomic names to label the nodes in this hierarchy. Taxonomic data is becoming increasingly available on the web, but scientists need a way to access it in a programmatic fashion that's easy and reproducible. We have developed taxize, an open-source software package (freely available from http://cran.r-

project.org/web/packages/taxize/index.html) for the R language. taxize provides simple, programmatic access to taxonomic data for 13 data sources around the web. We discuss the need for a taxonomic toolbelt in R, and outline a suite of use cases for which taxize is ideally suited (including a full workflow as an appendix). The taxize package facilitates open and reproducible science by allowing taxonomic data collection to be done in the open-source R platform.

D This article is included in the RPackage gateway.

This article is included in the Phylogenetics collection.

\section{Open Peer Review \\ Approval Status \\ 2 \\ 3 \\ version 2 \\ (update) \\ 28 Oct 2013 \\ version 1 \\ 18 Sep 2013

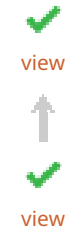

view
view

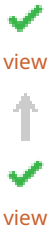

1. Will Pearse, University of Minnesota,

Minneapolis, MN, USA

2. Gavin L. Simpson, University of Regina,

Regina, SK, Canada

3. Ethan White (ID), Utah State University,

Logan, UT, USA

Any reports and responses or comments on the article can be found at the end of the article. 
Corresponding author: Scott A. Chamberlain (myrmecocystus@gmail.com)

Competing interests: No competing interests were disclosed.

Grant information: The author(s) declared that no grants were involved in supporting this work.

Copyright: ๑ 2013 Chamberlain SA and Szöcs E. This is an open access article distributed under the terms of the Creative Commons Attribution License, which permits unrestricted use, distribution, and reproduction in any medium, provided the original work is properly cited. Data associated with the article are available under the terms of the Creative Commons Zero "No rights reserved" data waiver (CC0 1.0 Public domain dedication).

How to cite this article: Chamberlain SA and Szöcs E. taxize: taxonomic search and retrieval in R [version 1; peer review: 3 approved] F1000Research 2013, 2:191 https://doi.org/10.12688/f1000research.2-191.v1

First published: 18 Sep 2013, 2:191 https://doi.org/10.12688/f1000research.2-191.v1 


\section{Introduction}

Evolution by natural selection has led to a hierarchical relationship among all living organisms. Thus, species are categorized using a taxonomic hierarchy, starting with the binomial species name (e.g, Homo sapiens), moving up to genus (Homo), then family (Hominidae), and on up to Domain (Eukarya). Biologists, whether studying organisms at the cell, organismal, or community level, can put their study objects into taxonomic context, allowing them to know close and distant relatives, find relevant literature, and more.

The use of taxonomic names is, unfortunately, not straightforward. Taxonomic names often vary due to name revisions at the generic or specific levels, lumping or splitting lower taxa (genera, species) among higher taxa (families), and name spelling changes. For example, a study found that a compilation of 308,000 plant observations from 51 digitized herbarium records had 22,100 unique taxon names, of which only 13,000 were accepted names ${ }^{1,2}$. In addition, there is no one authoritative taxonomic names source for all taxa - although, there are taxon specific sources that are used by many scientists. Different sources (e.g., uBio, Tropicos, ITIS) may use different accepted names for the same taxon. For example, while the Integrated Taxonomic Information Service (ITIS) has Helianthus $x$ glaucus as an accepted name, The Plant List (http://www.theplantlist.org) gives that name as unresolved. But Helianthus glaucus is an accepted name in The Plant List, while ITIS does not list this name.

One attempt to help inconsistencies in taxonomy is the use of numeric codes. For example, ITIS assigns a Taxonomic Serial Number (TSN) to each taxon, while the Universal Biological Indexer and Organizer (uBio) assigns each taxon a NameBank identifier (namebankID), and Tropicos assigns their own identifier to each taxon. Codes are helpful within a database as they can easily refer to, for example, Helianthus annuus with a code like 123456 instead of its whole name. However, each database uses their own code; in this case for Helianthus annuus, ITIS uses 36616, uBio uses 2658020, and Tropicos uses 40022652. As there are no universal codes for taxa across databases, this can lead to additional confusion. Last, name comparisons across databases have to be done with the actual names, not the codes.

Taxonomic data is getting easier to obtain through the web (e.g., http://eol.org/). However, there are a number of good reasons to obtain taxonomic information programatically rather than through a web interface. First, if you have more than a few names to look up on a website, it can take quite a long time to enter each name, get data, and repeat for each species. Programatically getting taxonomic names solves the problem by looping over a list of names. In addition, doing taxonomic searching, etc. becomes reproducible. With increasing reports of irreproducibility in science ${ }^{3,4}$, it is extremely important to make science workflows repeatable. Science workflows can now easily incorporate text, code, and images in a single executable document ${ }^{5}$. Reproducible documents should become mainstream in biology to avoid mistakes, and make collaboration easier.
The $\mathrm{R}$ language is widely used by biologists, and now has over 5,000 packages on the Comprehensive R Archive Network (CRAN) to extend R. R is great for manipulating, visualizing and fitting statistical models to data. Gentleman et al. ${ }^{6}$ give a detailed discussion of advantages of $\mathrm{R}$ in computational biology. Getting data from the web will be increasingly common as more and more data gets moved to the cloud. Therefore there is a need to get data from the web directly into R. Increasingly, data is available from the web via application programming interfaces (API). These allow computers to talk to one another using code that is not human readable, but is machine readable. Web APIs often define a number of methods that allow users to search for a species name, or retrieve the synonyms for a species name, for example. A further advantage of APIs is that they are language agnostic, meaning that data can be consumed in almost any computing context, allowing users to interact with the web API without having to know the details of the code. Moreover data can be accessed from every computer, whereas for example an Excel file can only be opened in a few programs.

The goal of taxize, an R package in development, is to make many use cases that involve retrieving and resolving taxonomic names easy and reproducible. In taxize, we have written a suite of R functions that interact with many taxonomic data sources via their web APIs (Table 1). The interface to each function is usually a simple list of species names, just as a user would enter when interacting with a website. Therefore, we hope that moving from a web to an $\mathrm{R}$ interface for taxonomic names will be relatively seamless (if one is already nominally familiar with $\mathrm{R}$ ).

Here, we justify the need for programmatic taxonomic resolution tools like taxize, discuss our data sources, and run through a suite of use cases to demonstrate the variety of ways that users can use taxize.

\section{Why do we need taxize?}

There is a large suite of applications developed around the problem of searching for, resolving, and getting higher taxonomy for species names. For example, Linnaeus http://linnaeus.sourceforge.net/ provides the ability to search for taxonomic names in documents and normalize those names found. In addition, there are many web interfaces to search for and normalize names such as Encyclopedia of Life's Global Names Resolver http://resolver.globalnames.org/, uBio tools http://www.ubio.org/index.php?pagename=sample_ tools, and iPlant's Taxonomic Name Resolution Service http://tnrs. iplantcollaborative.org/.

All of these data repositories provide ways to search for taxonomic names and resolve them in some cases. However, scientists ideally need a tool that is free and can be used programmatically, thereby facilitating reproducible research. The goal of taxize is to facilitate the creation of reproducible and easy to use workflows for searching for taxonomic names, resolving them, getting higher taxonomic names, and other tasks related to research dealing with species.

\section{Data sources and package details}

taxize uses many data sources (Table 1), and more can easily be added. There are two common tasks provided by the data sources: 
Table 1. Some key functions in taxize, what they do, and their data sources.

\begin{tabular}{|c|c|c|}
\hline Function name & What it does & Source \\
\hline apg_lookup & Changes names to match the APGIII list & $\begin{array}{l}\text { Angiosperm Phylogeny Group http://www.mobot.org/MOBOT/ } \\
\text { research/APweb/ }\end{array}$ \\
\hline classification & Upstream classification & Various \\
\hline col_children & Direct children & Catalogue of Life http://www.catalogueoflife.org/ \\
\hline col_downstream & Downstream taxa to specified rank & Catalogue of Life http://www.catalogueoflife.org/ \\
\hline eol_hierarchy & Upstream classification & Encyclopedia of Life http://eol.org/ \\
\hline eol_search & Search EOL taxon information & Encyclopedia of Life http://eol.org/ \\
\hline get_seqs & Get NCBI sequences & National Center for Biotechnology Information federhen \\
\hline get_tsn & Get ITIS TSN & Integrated Taxonomic Information System http://www.itis.gov/ \\
\hline get_uid & Get NCBI UID & National Center for Biotechnology Information ${ }^{7}$ \\
\hline searchbycommonname & Search ITIS by common name & Integrated Taxonomic Information System http://www.itis.gov/ \\
\hline searchbyscientificname & Search ITIS by scientific name & Integrated Taxonomic Information System http://www.itis.gov/ \\
\hline gisd_isinvasive & Invasiveness status & $\begin{array}{l}\text { Global Invasive Species Database http://www.issg.org/database/ } \\
\text { welcome/ }\end{array}$ \\
\hline gni_parse & Parse scientific names into components & Global Names Index http://gni.globalnames.org/ \\
\hline gni_search & Search EOL's global names index & Global Names Index http://gni.globalnames.org/ \\
\hline gnr_resolve & $\begin{array}{l}\text { Resolve names using EOL's global } \\
\text { names index }\end{array}$ & Global Names Resolver http://resolver.globalnames.org/ \\
\hline itis_downstream & Downstream taxa to specified rank & Integrated Taxonomic Information System http://www.itis.gov/ \\
\hline iucn_status & IUCN status & IUCN Red List http://www.iucnredlist.org \\
\hline phylomatic_tree & Get a plant Phylogeny & Phylomatic $^{8}$ \\
\hline plantminer & Search Plantminer & Plantminer $^{9}$ \\
\hline tax_name & Get taxonomic name for specific rank & Various \\
\hline tax_rank & Get rank of a taxonomic name & Various \\
\hline tnrs & Resolve names using iPlant & $\begin{array}{l}\text { iPlant Taxonomic Name Resolution Service http://tnrs. } \\
\text { iplantcollaborative.org/ }\end{array}$ \\
\hline tp_acceptednames & Check for accepted names using Tropicos & Tropicos http://www.tropicos.org/ \\
\hline tpl_search & Search the Plant List & The Plant List http://www.theplantlist.org \\
\hline ubio_namebank & Search uBio & uBio http://www.ubio.org/index.php?pagename=sample_tools \\
\hline
\end{tabular}

name search and name resolution. Other functionality in taxize includes retrieving a classification tree for a species, or retrieving child taxa of a focal taxon. One of the data sources (Phylomatic) returns phylogenies, while another (NCBI) returns genetic sequence data. However, there are other $\mathrm{R}$ packages that are focused solely on sequence data, such as rsnps ${ }^{10}$, rentrez $z^{11}$, BoSSA $^{12}$, and ape ${ }^{13}$, so taxize does not venture deeply into these other domains.

Some of the data sources taxize interacts with require authentication. That is, in addition to the search terms the user provides (e.g., Homo sapiens), the data provider requires an alphanumeric identification key so that they can better manage their servers, collect analytics, and shut down users that abuse the API. The services that require an API key in taxize are: Encyclopedia of Life (EOL) http://eol.org/, the Universal Biological Indexer and Organizer (uBio) http://www.ubio. org/index.php?pagename=sample_tools, Tropicos http://www.tropicos.org/, and Plantminer ${ }^{9}$. One can easily obtain API keys by visiting the website of each service (see Table 1 for links to each site). There are two typical ways of using API keys. First, you can pass in your API key in a function call (e.g., ubio_namebank(srchName='Ursus americanus', key='your_alphanumeric_key')). Second, you can store your key in the .Rprofile file, which is a common place to store settings. We recommend the second option as it simplifies function calls as taxize detects the stored keys.

One available data source in taxize is The Plant List http://www. theplantlist.org. The connection in taxize is done via the taxonstand package $^{14,15}$ that solely interacts with The Plant List. We provide a few convenience functions that wrap taxonstand into taxize.

taxize would not have been possible without the work of others. taxize uses httr ${ }^{16}$ and RCurl $^{17}$ for performing calls to web APIs, XML ${ }^{18}$ for parsing XML, RJSONIO ${ }^{19}$ for parsing JSON, and $\operatorname{stringr}^{20}$ and $\mathrm{plyr}^{21}$ for manipulating data.

New data sources can be added; for example, we plan to add the following sources: Wikispecies and The Tree of Life. A connection to www.freshwaterecology.info (a database with autecological characteristics, ecological preferences and biological traits as well as distribution patterns of more than 12,000 European freshwater organisms belonging to fish, macro-invertebrates, macrophytes, diatoms and phytoplankton) will be finished when their new API is released. In addition, the authors welcome further suggestions of data sources to be added. 


\section{Use cases}

First, install taxize

First, one must install and load taxize into the R session.

install.packages ("taxize")

library (taxize)

Advanced users can also download and install the latest development copy from GitHub http://github.com/ropensci/taxize_, also permanently available at http://dx.doi.org/10.5281/zenodo.7097.

\section{Resolve taxonomic names}

This is a common task in biology. We often have a list of species names and we want to know a) if we have the most up to date names, b) if our names are spelled correctly, and c) the scientific name for a common name. One way to resolve names is via the Global Names Resolver (GNR) service provided by the Encyclopedia of Life http://resolver.globalnames.org/. Here, we are searching for two misspelled names:

\begin{tabular}{|c|c|c|c|}
\hline & matched name & & data_source_title \\
\hline 1 & Helianthus annuūs $\mathrm{L}$. & & Catalogue of Life \\
\hline 2 & Helianthus annus & GBIF & Taxonomic Backbone \\
\hline 3 & Helianthus annus & & EOL \\
\hline 4 & Helianthus annuus $\mathrm{L}$. & & EOL \\
\hline 5 & Helianthus annus & & uBio NameBank \\
\hline Homo & sapiens Linnaeus, 1758 & & Catalogue of Life \\
\hline
\end{tabular}

The correct spellings are Helianthus annuus and Homo sapiens. Another approach uses the Taxonomic Name Resolution Service via the Taxosaurus API http://taxosaurus.org/ developed by iPLant and the Phylotastic organization. In this example, we provide a list of species names, some of which are misspelled, and we'll call the API with the tnrs function.

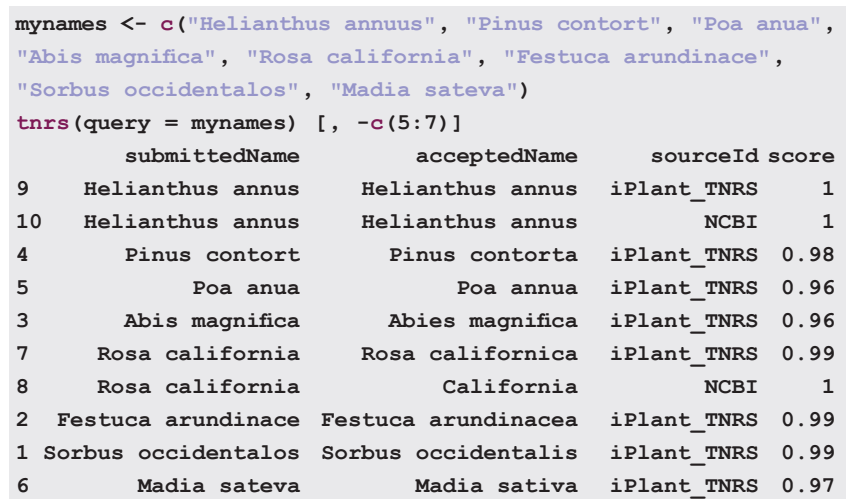

It turns out there are a few corrections: e.g., Madia sateva should be Madia sativa, and Rosa california should be Rosa californica. Note that this search worked because fuzzy matching was employed to retrieve names that were close, but not exact matches. Fuzzy matching is only available for plants in the TNRS service, so we advise using EOL's Global Names Resolver if you need to resolve animal names. taxize takes the approach that the user should be able to make decisions about what resource to trust, rather than making the decision on behalf of the user. Both the EOL GNR and the TNRS services provide data from a variety of data sources. The user may trust a specific data source, and thus may want to use the names from that data source. In the future, we may provide the ability for taxize to suggest the best match from a variety of sources.

Another common use case is when there are many synonyms for a species. In this example, we have six synonyms of the currently accepted name for a species.

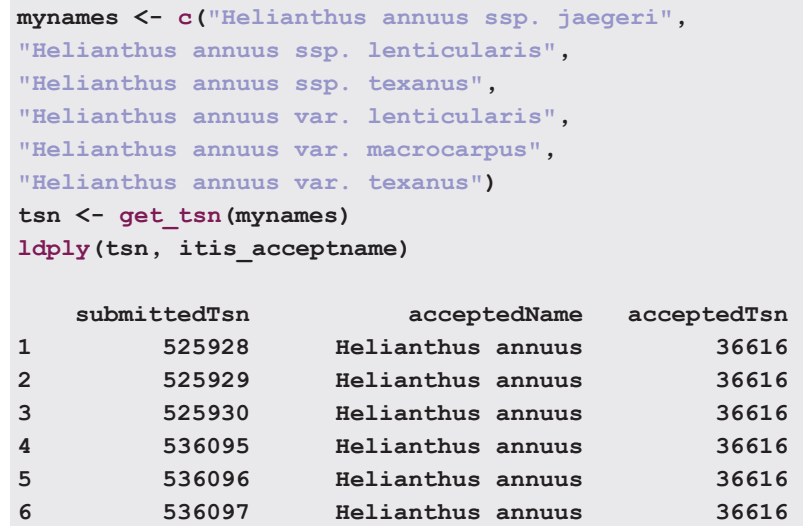

Retrieve higher taxonomic names

Another task biologists often face is getting higher taxonomic names for a taxa list. Having the higher taxonomy allows you to put into context the relationships of your species list. For example, you may find out that species A and species B are in Family C, which may lead to some interesting insight, as opposed to not knowing that Species A and B are closely related. This also makes it easy to aggregate/standardize data to a specific taxonomic level (e.g., family level) or to match data to other databases with different taxonomic resolution (e.g., trait databases).

A number of data sources in taxize provide the capability to retrieve higher taxonomic names, but we will highlight two of the more useful ones: Integrated Taxonomic Information System (ITIS) http:// www.itis.gov/ and National Center for Biotechnology Information (NCBI) ${ }^{7}$. First, we'll search for two species, Abies procera and Pinus contorta within ITIS.

\begin{tabular}{|c|c|c|c|}
\hline \multicolumn{4}{|c|}{ \$'Abies procera' } \\
\hline & rankName & taxonName & tsn \\
\hline 1 & Kingdom & Plantae & 202422 \\
\hline 2 & Subkingdom & Viridaeplantae & 846492 \\
\hline 3 & Infrakingdom & Streptophyta & 846494 \\
\hline 4 & Division & Tracheophyta & 846496 \\
\hline 5 & Subdivision & Spermatophytina & 846504 \\
\hline 6 & Infradivision & Gymnospermae & 846506 \\
\hline 7 & Class & Pinopsida & 500009 \\
\hline 8 & Order & Pinales & 500028 \\
\hline 9 & Family & Pinaceae & 18030 \\
\hline 10 & Genus & Abies & 18031 \\
\hline 11 & Species & Abies procera & 181835 \\
\hline
\end{tabular}




$\begin{array}{lrrr}\text { \$'Pinus contorta' } & & \\ & \text { rankName } & \text { taxonName } & \text { tsn } \\ 1 & \text { Kingdom } & \text { Plantae } & 202422 \\ 2 & \text { Subkingdom } & \text { Viridaeplantae } & 846492 \\ 3 & \text { Infrakingdom } & \text { Streptophyta } & 846494 \\ 4 & \text { Division } & \text { Tracheophyta } & 846496 \\ 5 & \text { Subdivision } & \text { Spermatophytina } & 846504 \\ 6 & \text { Infradivision } & \text { Gymnospermae } & 846506 \\ 7 & \text { Class } & \text { Pinopsida } & 500009 \\ 8 & \text { Order } & \text { Pinales } & 500028 \\ 9 & \text { Family } & \text { Pinaceae } & 18030 \\ 10 & \text { Genus } & \text { Pinus } & 18035 \\ 11 & \text { Species } & \text { Pinus contorta } & 183327\end{array}$

It turns out both species are in the family Pinaceae. You can also get this type of information from the NCBI by excuting the following code in R: classification (specieslist, $d b=$ 'ncbi').

Instead of a full classification, you may only want a single name, say a family name for your species of interest. The function tax_name is built just for this purpose. As with the classificationfunction you can specify the data source with the $d b$ argument, either ITIS or NCBI.

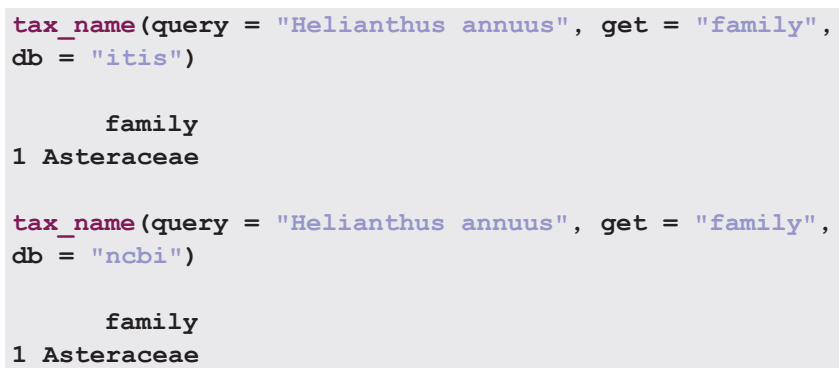

If a data source does not provide information on the queried species, the result could be taken from another source and the results from the different sources could be pooled.

\section{Interactive name selection}

As mentioned previously most databases use a numeric code to reference a species. A general workflow in taxize is: Retrieve Code for the queried species and then use this code to query more data/information. Below are a few examples. When you run these examples in $\mathrm{R}$, you are presented with a command prompt asking for the row that contains the name you would like back; that output is not printed below for brevity. In this example, the search term has many matches. The function returns a data.frame of the matches, and asks for the user to input which row number to accept.

\begin{tabular}{|c|c|c|}
\hline & combinedname & $\operatorname{tsn}$ \\
\hline 1 & Heliastes bicolor & 615238 \\
\hline 2 & Heliastes chrysurus & 615250 \\
\hline 3 & Heliastes cinctus & 615573 \\
\hline 4 & Heliastes dimidiatus & 615257 \\
\hline 5 & Heliastes hypsilepis & 615273 \\
\hline 6 & Heliastes immaculatus & 615639 \\
\hline 7 & Heliastes opercularis & 615300 \\
\hline 8 & Heliastes ovalis & 615301 \\
\hline $\begin{array}{l}\text { Nz } \\
\text { at } \\
{[]}\end{array}$ & $\begin{array}{l}\text { class") } \\
\text { "n" }\end{array}$ & \\
\hline
\end{tabular}

In another example, you can pass in a long character vector of taxonomic names:

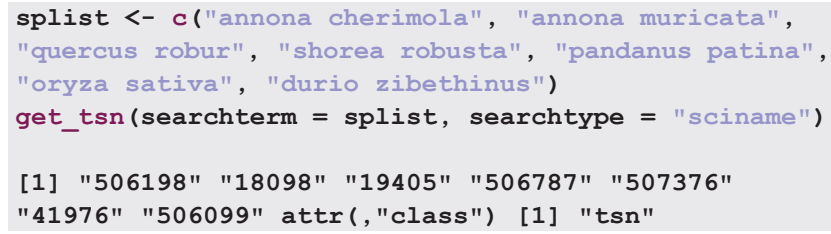

In another example, note that no match at all returns an NA:

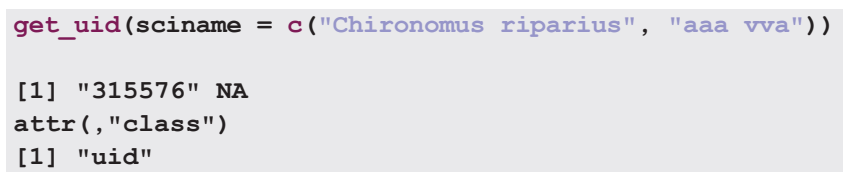

\section{Retrieve a phylogeny}

Ecologists are increasingly taking a phylogenetic approach to ecology, applying phylogenies to topics such as the study of community structure $^{22}$, ecological networks ${ }^{23}$, and functional trait ecology ${ }^{24}$. Yet, many biologists are not adequately trained in reconstructing phylogenies. Fortunately, there are some sources for getting a phylogeny without having to know how to build one; one of these is for angiosperms, called Phylomatic ${ }^{8}$. We have created a workflow in taxize that accepts a species list, and taxize works behind the scenes to get higher taxonomic names, which are required by Phylomatic to get a phylogeny. Here is a short example, producing the tree in Figure 1.

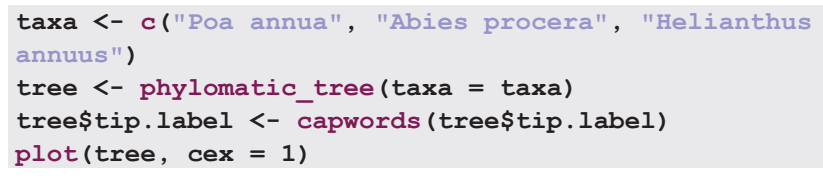

Behind the scenes the function phylomatic_tree retrieves a Taxonomic Serial Number (TSN) from ITIS for each species name, then a string is created for each species like this poaceaeloryza/oryza_ sativa (with format "family/genus/genus_epithet"). These strings are submitted to the Phylomatic API, and if no errors occur, a phylogeny in newick format is returned. The phylomatic_tree() function also cleans up the newick string and converts it to an ape phylo object. The output from phylomatic_tree() is a phylo object, which can be used for plotting and phylogenetic analyses. Be aware that Phylomatic has certain limitations - refer to the paper describing Phylomatic ${ }^{8}$ and the website http://phylodiversity.net/phylomatic/.

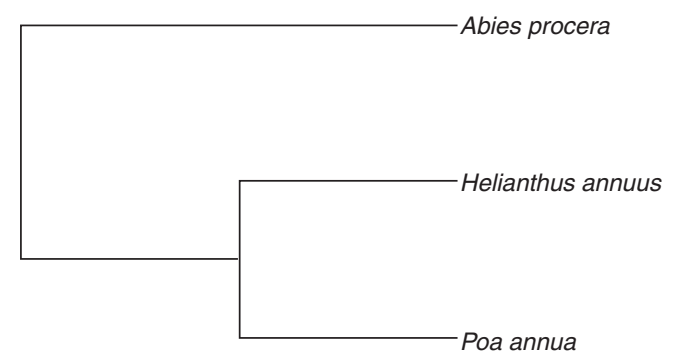

Figure 1. A phylogeny for three species. This phylogeny was produced using the phylomatic tree function, which queries the Phylomatic database, and prunes a previously created phylogeny of plants. 
There are currently no resources for getting a phylogeny of animals simply from species names. However, a few projects are working on this problem, including the Open Tree of Life http://blog.opentreeoflife.org/. We will incorporate these resources when the appropriate APIs are available.

\section{What taxa are the children of my taxon of interest?}

If someone is not a taxonomic specialist on a particular taxon they probably do not know what children taxa are within a family, or within a genus. This task becomes especially unwieldy when there are a large number of taxa downstream. You can of course go to a website like Wikispecies http://species.wikimedia.org/wiki/Main_Page or Encyclopedia of Life http://eol.org/ to get downstream names. However, taxize provides an easy way to programatically search for downstream taxa, both for the Catalogue of Life (CoL) http://www. catalogueoflife.org/ and the Integrated Taxonomic Information System http://www.itis.gov/. Here is a short example using the CoL in which we want to find all the species within the genus.

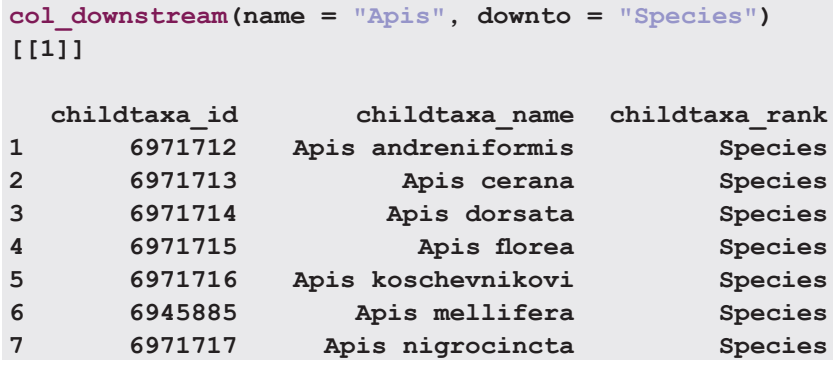

childtaxa rank Species Species Species Species Species Species Species

The result from the above call to col_downstream() is a data.frame that gives a number of columns of different information.

\section{IUCN status}

There are a number of things we can do once we have the correct taxonomic names. One thing we can do is ask about the conservation status of a species (IUCN Red List of Threatened Species http://www.iucnredlist.org). We have provided a set of functions, iucn_summary and iucn_status, to search for species names, and extract the status information, respectively. Here, we search for the panther and lynx.

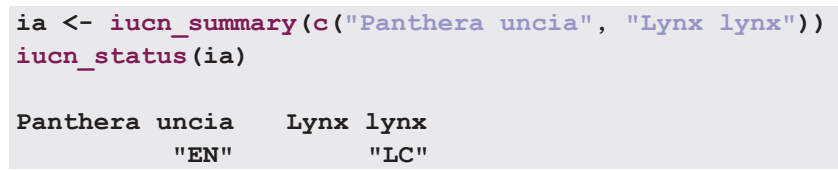

It turns out that the panther has a status of endangered (EN) and the lynx has a status of least concern (LC).

\section{Search for available genes in GenBank}

Another use case available in taxize deals with genetic sequences. taxize has three functions to interact with GenBank to search for available genes (get_genes_avail), download genes by GenBank ID (get_genes), and download genes via taxonomic name search, including retrieving a congeneric if the searched taxon does not exist in the database (get_seqs). In this example, we search for gene sequences for Umbra limi. out <- get_genes_avail(taxon_name = "Umbra limi", seqrange $=" 1: 20 \overline{0} 0 "$, getrelated = FALSE)

Then we can ask if 'RAG1' exists in any of the gene names.

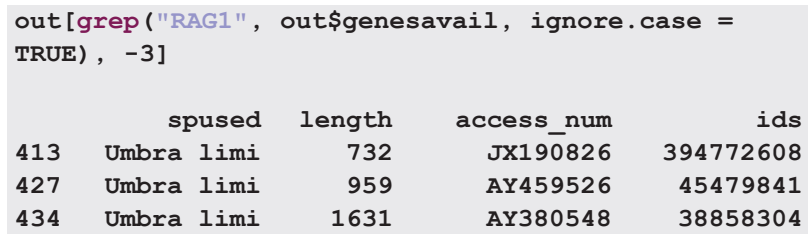

It turns out that there are 430 different unique records found. However, this doesn't mean that there are 430 different genes found as the API does not provide metadata to classify genes. However, you can use regular expressions (e.g., grep) to search for the gene of interest.

Matching species tables with different taxonomic resolution Biologists often need to match different sets of data tied to species. For example, trait-based approaches are a promising tool in ecology ${ }^{25}$. One problem is that abundance data must be matched with trait databases such as the NCBI Taxonomy database ${ }^{26}$. These two data tables may contain species information on different taxonomic levels and data might have to be aggregated to a joint taxonomic level, so that the data can be merged. taxize can help in this data-cleaning step, providing a reproducible workflow.

We can use the mentioned classification-function to retrieve the taxonomic hierarchy and then search the hierarchies up- and downwards for matches. Here is an example to match a species with names on three different taxonomic levels.

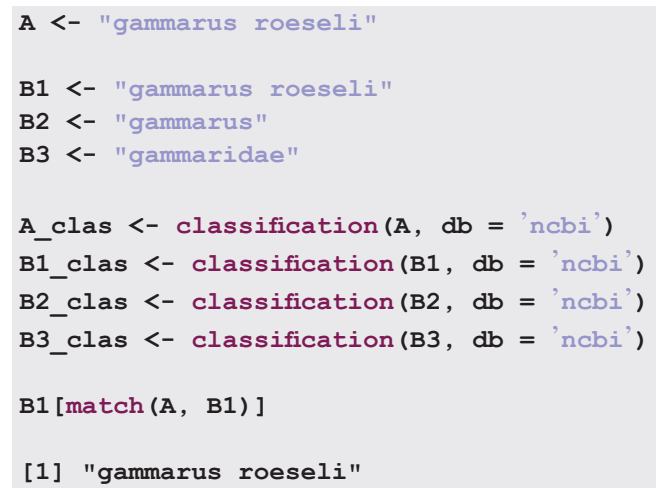

If we find a direct match (here Gammarus roeseli), we are lucky. But we can also match Gammaridae with Gammarus roeseli, but on a lower taxonomic level. A more comprehensive and realistic 
example (matching a trait table with an abundance table) is given in Appendix B.

\section{Aggregating data to a specific taxonomic rank}

In biology, one can asks questions at varying taxonomic levels. This use case is easily handled in taxize. A function called tax agg will aggregate community data to a specific taxonomic level. In this example, we take the data for three species and aggregate them to family level. Again we can specify if we want to use data from ITIS or NCBI. The rows in the data.frame are different communities.

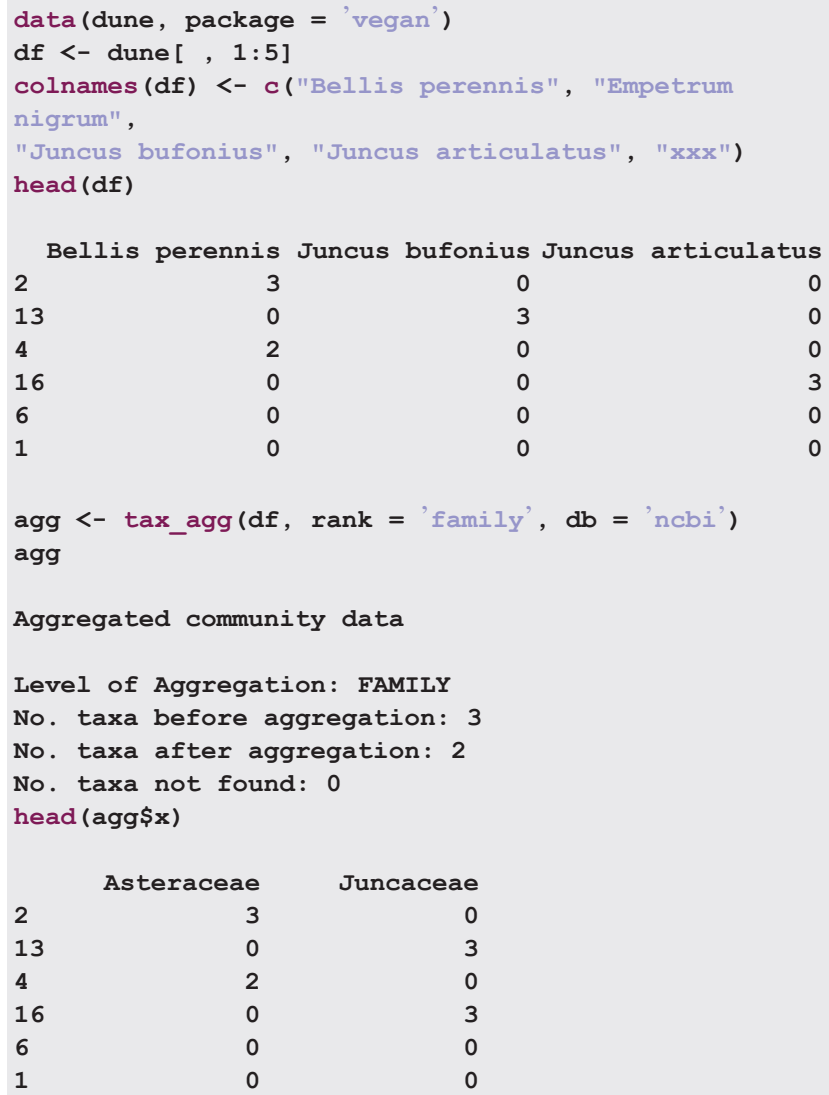

$\begin{array}{lrr} & \text { Asteraceae } & \text { Juncaceae } \\ 2 & 3 & 0 \\ 13 & 0 & 3 \\ 4 & 2 & 0 \\ 16 & 0 & 3 \\ 6 & 0 & 0 \\ 1 & 0 & 0\end{array}$

The two Juncus species are aggregated to the family Juncaceae and their abundances are summed. There was only a single species in the family Asteraceae, so the data for Bellis perennis are carried over.

\section{Conclusions}

Taxonomic information is increasingly sought out by biologists as we take phylogenetic and taxonomic approaches to science. Taxonomic data are becoming more widely available on the web, yet scientists require programmatic access to this data for developing reproducible workflows. taxize was created to bridge this gap - to bring taxonomic data on the web into $\mathrm{R}$, where the data can be easily manipulated, visualized, and analyzed in a reproducible workflow.

We have outlined a suite of use cases in taxize that will likely fit real use cases for many biologists. Of course we have not thought of all possible use cases, so we hope that the biology community can give us feedback on what use cases they want to see available in taxize. One thing we could change in the future is to make functions that fit use cases, and then allow users to select the data source as a parameter in the function. This could possibly make the user interface easier to understand.

taxize is currently under development and will be for some time given the large number of data sources knitted together in the package, and the fact that APIs for each data source can change, requiring changes in taxize code. Contributions to taxize are strongly encouraged, and can be easily done using GitHub here http://github. com/ropensci/taxize_. We hope taxize will be taken up by the community and developed collaboratively, making it progressively better through time as new use cases arise, bug reports are squashed, and contributions are merged.

\section{Author contributions}

SC and ES equally contributed to the software discussed in this paper, and contributed equally to writing of the manuscript.

\section{Competing interests}

No competing interests were disclosed.

\section{Grant information}

The author(s) declared that no grants were involved in supporting this work.

\section{Acknowledgements}

The taxize package is part of the rOpenSci project http://ropensci.org/. We thank Carl Boettiger, Karthik Ram, Owen Jones, Naim Matasci, and Ralf Schäfer for comments on previous versions of this manuscript. We thank all API maintainers for their work making their databases open to the public. 
1. Weiser MD, Enquist BJ, Boyle B, et al.: Latitudinal patterns of range size and species richness of new world woody plants. Global Ecology and Biogeography. 2007; 16(5): 679-688.

Publisher Full Text

2. Boyle B, Hopkins N, Lu Z, et al: The taxonomic name resolution service: an online tool for automated standardization of plant names. BMC Bioinformatics. 2013; 14(1): 16.

PubMed Abstract | Publisher Full Text | Free Full Text

3. Stodden VC: Reproducible research: Addressing the need for data and code sharing in computational science. Comput Sci Eng. 2010; 12(5): 8-13. Publisher Full Text

4. Zimmer C: A sharp rise in retractions prompts calls for reform. New York Times. 2012. Reference Source

5. Xie Y: Dynamic Documents with R and knitr. Chapman and Hall/CRC, 2013. Reference Source

6. Gentleman RC, Carey VJ, Bates DM, et al: Bioconductor: open software development for computational biology and bioinformatics. Genome Biol. 2004; 5(10): R80. PubMed Abstract | Publisher Full Text | Free Full Text

7. Federhen S: The ncbi taxonomy database. Nucleic Acids Res. 2012; 40 (Database issue): D136-D143.

PubMed Abstract | Publisher Full Text | Free Full Text

8. Webb CO, Donoghue MJ: Phylomatic: tree assembly for applied phylogenetics. Mol Ecol Notes. 2005; 5(1): 181-183.

Publisher Full Text

9. Carvalho GH, Cianciaruso MV, Batalha MA: Plantminer: a web tool for checking and gathering plant species taxonomic information. Environmental Modelling \& Software. 2010; 25(6): 815-816. Publisher Full Text

10. Chamberlain S, Ushey K: rsnps: Interface to SNP data on the web. $R$ package version 0.0 .42013 Reference Source

11. Winter D: rentrez: Entrez in R, R package version 0.2.12013. Reference Source

12. Lefeuvre P: BoSSA: a Bunch of Structure and Sequence Analysis, R package version 1.22010 Reference Source

13. Paradis E, Claude J, Strimmer K: APE: analyses of phylogenetics and evolution in R language. Bioinformatics. 2004; 20(2): 289-290. PubMed Abstract | Publisher Full Text

14. Cayuela L, Granzow-de la Cerda I, Al-buquerque FS, et al:: taxonstand: An r package for species names standardisation in vegetation databases. Methods Ecol Evol. 2012; 3(6): 1078-1083. Publisher Full Text

15. Cayuela L: Taxonstand: Taxonomic standardization of plant species names. $R$ package version 1.02012 Reference Source
16. Wickham $\mathrm{H}$ : httr: Tools for working with URLs and HTTP. R package version 0.2 2012. Reference Source

17. Lang DT: RCurl: General network (HTTP/FTP/...) client interface for R. R package version 1.95-4.12013.

Reference Source

18. Lang DT: $\mathbf{X M L}$ : Tools for parsing and generating $\mathbf{X M L}$ within $\mathbf{R}$ and S-Plus. $R$ package version 3.95-0.2 2013.

Reference Source

19. Lang DT: RJSONIO: Serialize R objects to JSON, JavaScript Object Notation. R package version 1.0-3 2013 Reference Source

20. Wickham $\mathrm{H}$ : stringr: Make it easier to work with strings. $\mathrm{R}$ package version 0.6 .2 2012.

Reference Source

21. Wickham $\mathrm{H}$ : The split-apply-combine strategy for data analysis. J Stat Softw. 2011; 40(1): 1-29. Reference Source

22. Webb CO, Ackerly DD, McPeek MA, et al.: Phylogenies and community ecology. Annu Rev Ecol Syst. 2002; 33: 475-505. Publisher Full Text

23. Rafferty NE, Ives AR: Phylogenetic trait-based analyses of ecological networks. Ecology. 2013 Publisher Full Text

24. Poff NL, Olden JD, Vieira NKM, et al.: Functional trait niches of north american lotic insects: traits-based ecological applications in light of phylogenetic relationships. Journal of the North American Benthological Society. 2006; 25(4): 730-755.

Publisher Full Text

25. Statzner B, Bêche LA: Can biological invertebrate traits resolve effects of multiple stressors on running water ecosystems? Freshwater Biology. 2010 55(Supplement s1): 80-119. Publisher Full Text

26. Usseglio-Polatera $\mathrm{P}$, Bournaud $\mathrm{M}$, Richoux $\mathrm{P}$, et al:: Biological and ecological traits of benthic freshwater macroinvertebrates: relationships and definition of groups with similar traits. Freshw Biol. 2000 43(2): 175-205

Publisher Full Text

27. Baird DJ, Baker CJ, Brua RB, et al:: Toward a knowledge infrastructure for traitsbased ecological risk assessment. Integr Environ Assess Manag. 2011; 7(2): 209-215

PubMed Abstract | Publisher Full Text

28. Kleyer M, Dray S, Bello F, et al:: Assessing species and community functional responses to environmental gradients: which multivariate methods? Journal of Vegetation Science. 2012; 23(5): 805-821. Publisher Full Text 


\section{Appendix A. A COMPLETE REPRODUCIBLE WORKFLOW, FROM A SPECIES LIST TO A PHYLOGENY, AND DISTRIBUTION MAP.}

If you aren't familiar with a complete workflow in $\mathrm{R}$, it may be difficult to visualize the process. In R, everything is programmatic, so the whole workflow can be in one place, and be repeated whenever necessary. The following is a workflow for taxize, going from a species list to a phylogeny.

First, install taxize

install.packages ("taxize")

Then load it into $\mathrm{R}$

library (taxize)

Most of us will start out with a species list, something like the one below. Note that each of the names is spelled incorrectly.

splist <- c("Helanthus annuus", "Pinos contorta", "Collomia grandiflorra", "Abies magnificaa", "Rosa california", "Datura wrighti", "Mimulus bicolour", "Nicotiana glauca", "Maddia sativa",

"Bartlettia scapposa")

There are many ways to resolve taxonomic names in taxize. Of course, the ideal name resolver will do the work behind the scenes for you so that you don't have to do things like fuzzy matching. There are a few services in taxize like this we can choose from: the Global Names Resolver service from EOL (see function gnr_resolve) and the Taxonomic Name Resolution Service from iPlant (see function tnrs). In this case let's use the function tnrs.

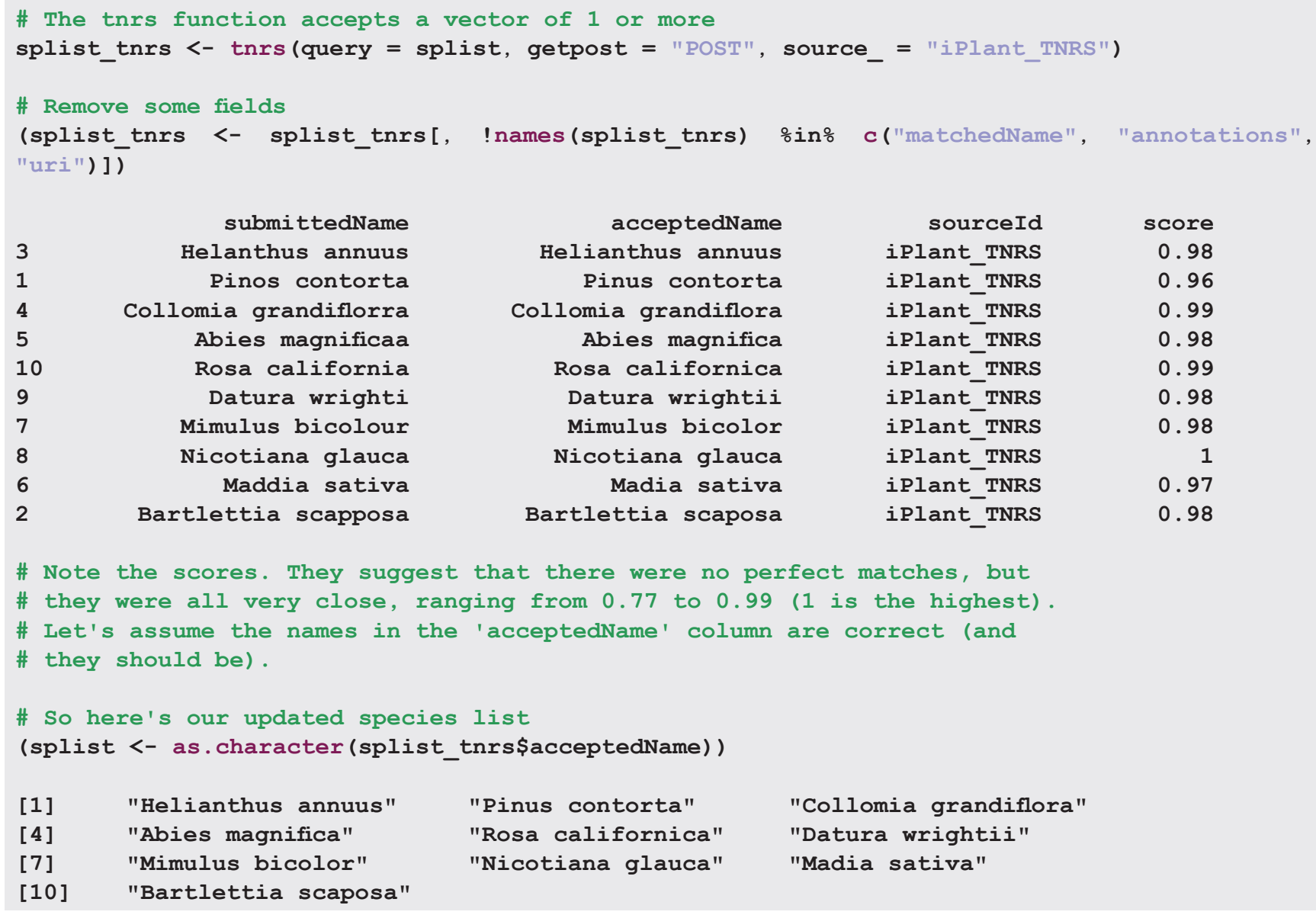

1

$\begin{array}{lr}\text { sourceId } & \text { score } \\ \text { iPlant_TNRS } & 0.98 \\ \text { iPlant_TNRS } & 0.96 \\ \text { iPlant_TNRS } & 0.99 \\ \text { iPlant_TNRS } & 0.98 \\ \text { iPlant_TNRS } & 0.99 \\ \text { iPlant_TNRS } & 0.98 \\ \text { iPlant_TNRS } & 0.98 \\ \text { iPlant_TNRS } & 1 \\ \text { iPlant_TNRS } & 0.97 \\ \text { iPlant_TNRS } & 0.98\end{array}$

\# Note the scores. They suggest that there were no perfect matches, but

\# they were all very close, ranging from 0.77 to 0.99 ( 1 is the highest).

\# Let's assume the names in the 'acceptedName' column are correct (and

\# they should be).

\# So here's our updated species list

(splist <- as.character (splist_tnrs\$acceptedName))

[1] "Helianthus annuus"

[4] "Abies magnifica"

[7] "Mimulus bicolor" "Nicotiana glauca"

"Pinus contorta"

"Rosa californica"

"Collomia grandiflora"

"Datura wrightii"

"Madia sativa"

"Bartlettia scaposa" 
Another thing we may want to do is collect common names for our taxa.

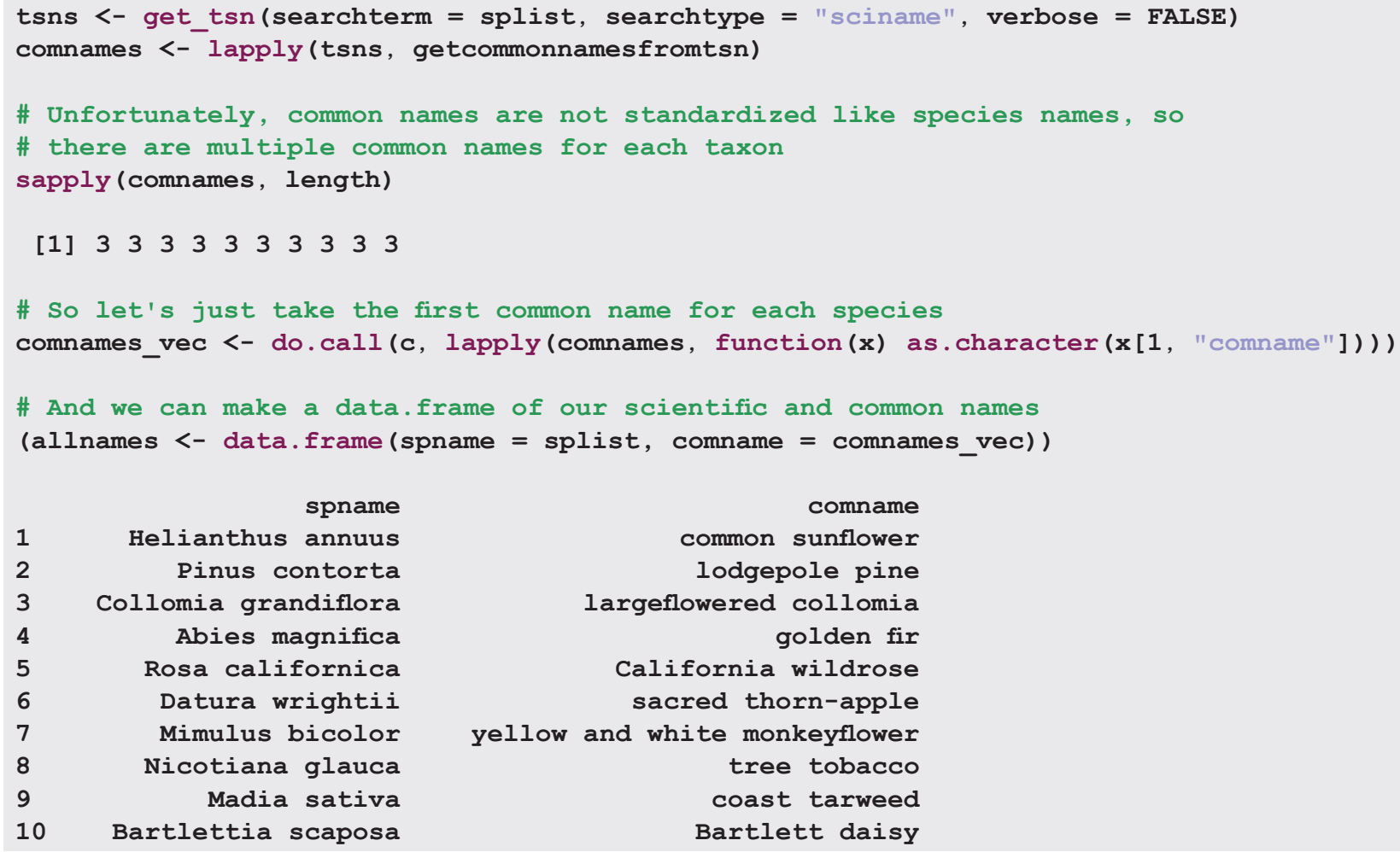

Another common task is getting the taxonomic tree upstream from your study taxa. We often know what family or order our taxa are in, but it we often don't know the tribes, subclasses, and superfamilies. taxize provides many avenues to getting classifications. Two of them are accessible via a single function (classification): the Integrated Taxonomic Information System (ITIS) and National Center for Biotechnology Information (NCBI); and via the Catalogue of Life (see function col classification):

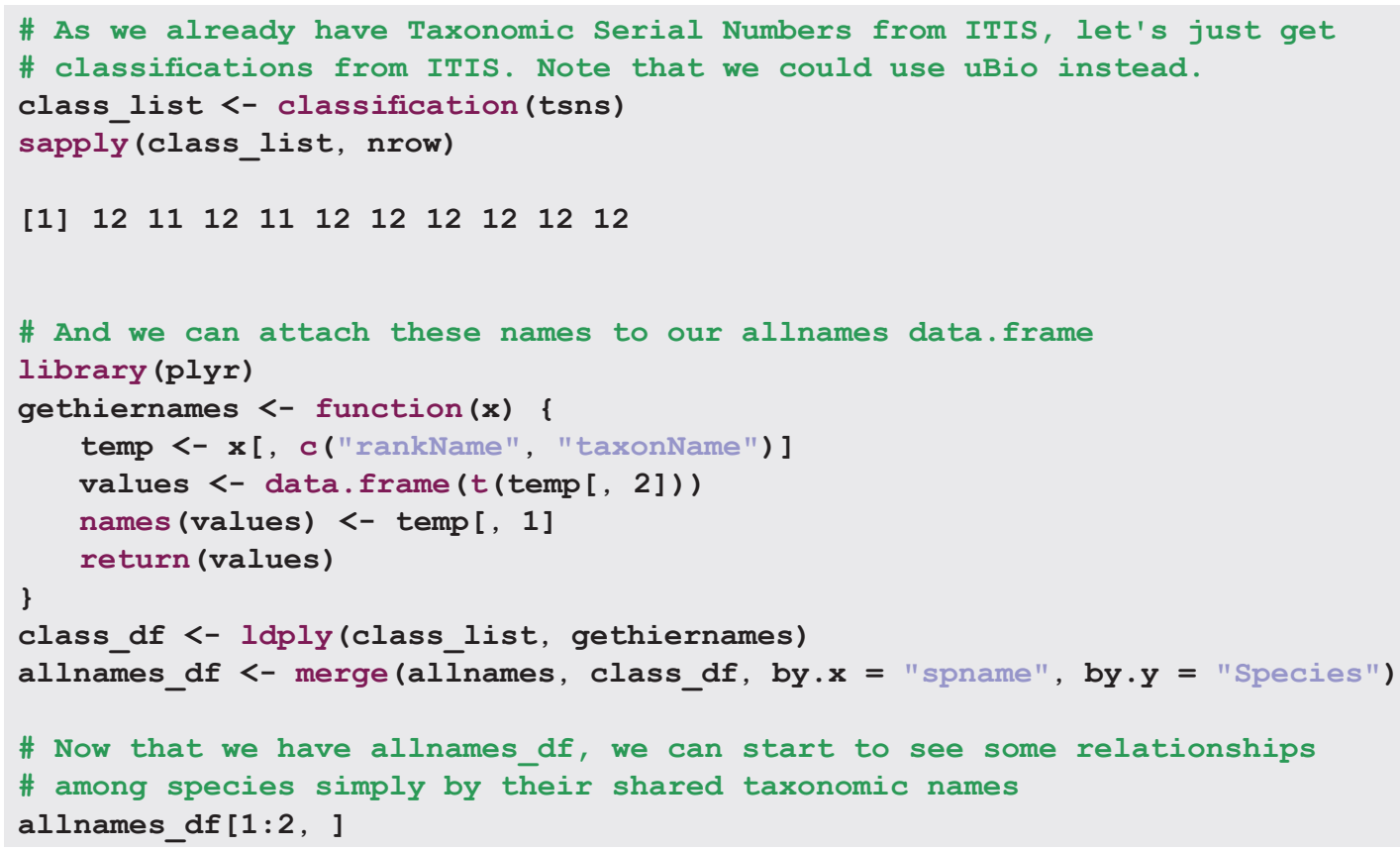




\begin{tabular}{|c|c|c|c|c|c|}
\hline & spname & comname & Kingdom & Subkingdom & Infrakingdom \\
\hline 1 & Abies magnifica & golden fir & Plantae & Viridaeplantae & streptophyta \\
\hline \multirow[t]{2}{*}{2} & Bartlettia scaposa & Bartlett daisy & Plantae & Viridaeplantae & streptophyta \\
\hline & Division & Subdivision & Infradivision & Class & Superorder \\
\hline 1 & Tracheophyta & Spermatophytina & Gymnospermae & Pinopsida & $<\mathrm{NA}>$ \\
\hline \multirow[t]{2}{*}{2} & Tracheophyta & Spermatophytina & Angiospermae & Magnoliopsida & Asteranae \\
\hline & Order & Family & Genus & & \\
\hline 1 & Pinales & Pinaceae & Abies & & \\
\hline 2 & Asterales & Asteraceae & Bartlettia & & \\
\hline
\end{tabular}

However, taxonomy can only get you so far. Shared ancestry can be reconstructed from molecular data, and phylogenies created. Phylomatic is a web service with an API that we can use to get a phylogeny.

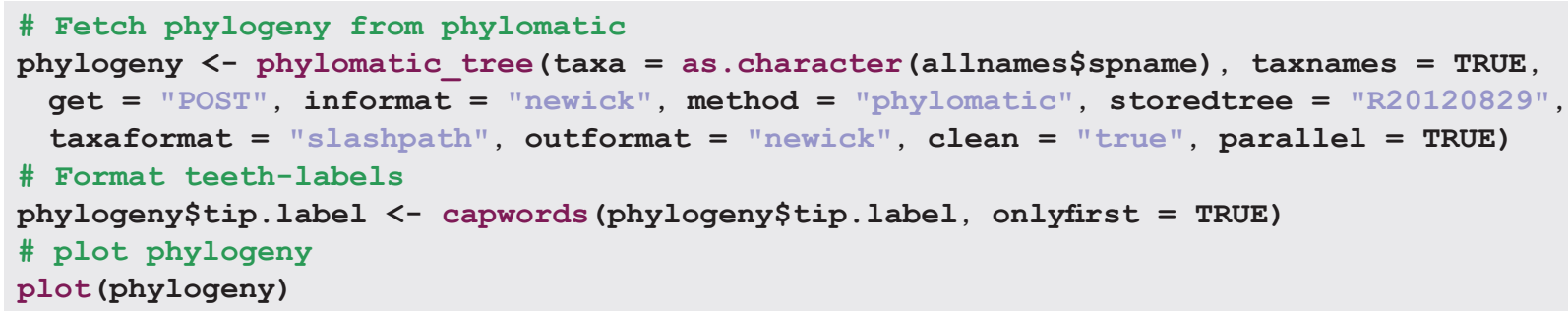

Using the species list, with the corrected names, we can now search for occurrence data. The Global Biodiversity Information Facility (GBIF) has the largest collection of records data, and has a API that we can interact with programmatically from R. First, we need to install rgbif.

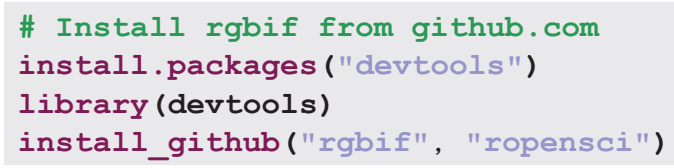

Now we can search for occurrences for our species list and make a map.

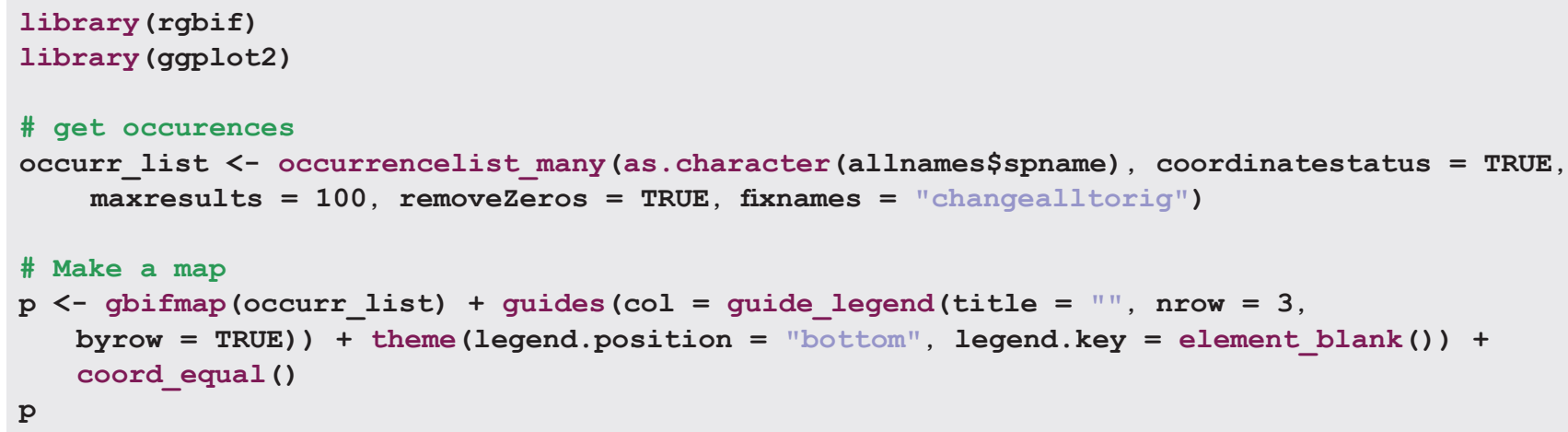




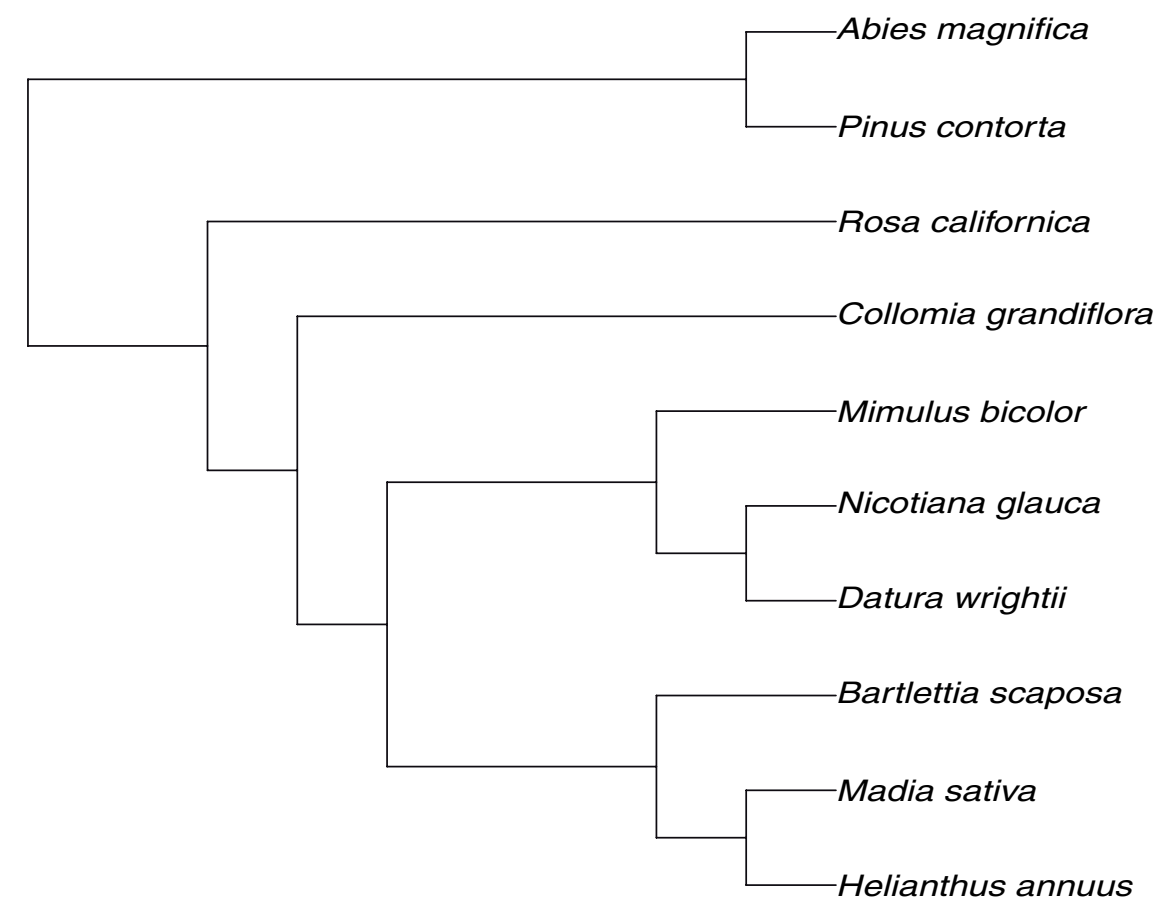

Figure 1. A phylogeny. 


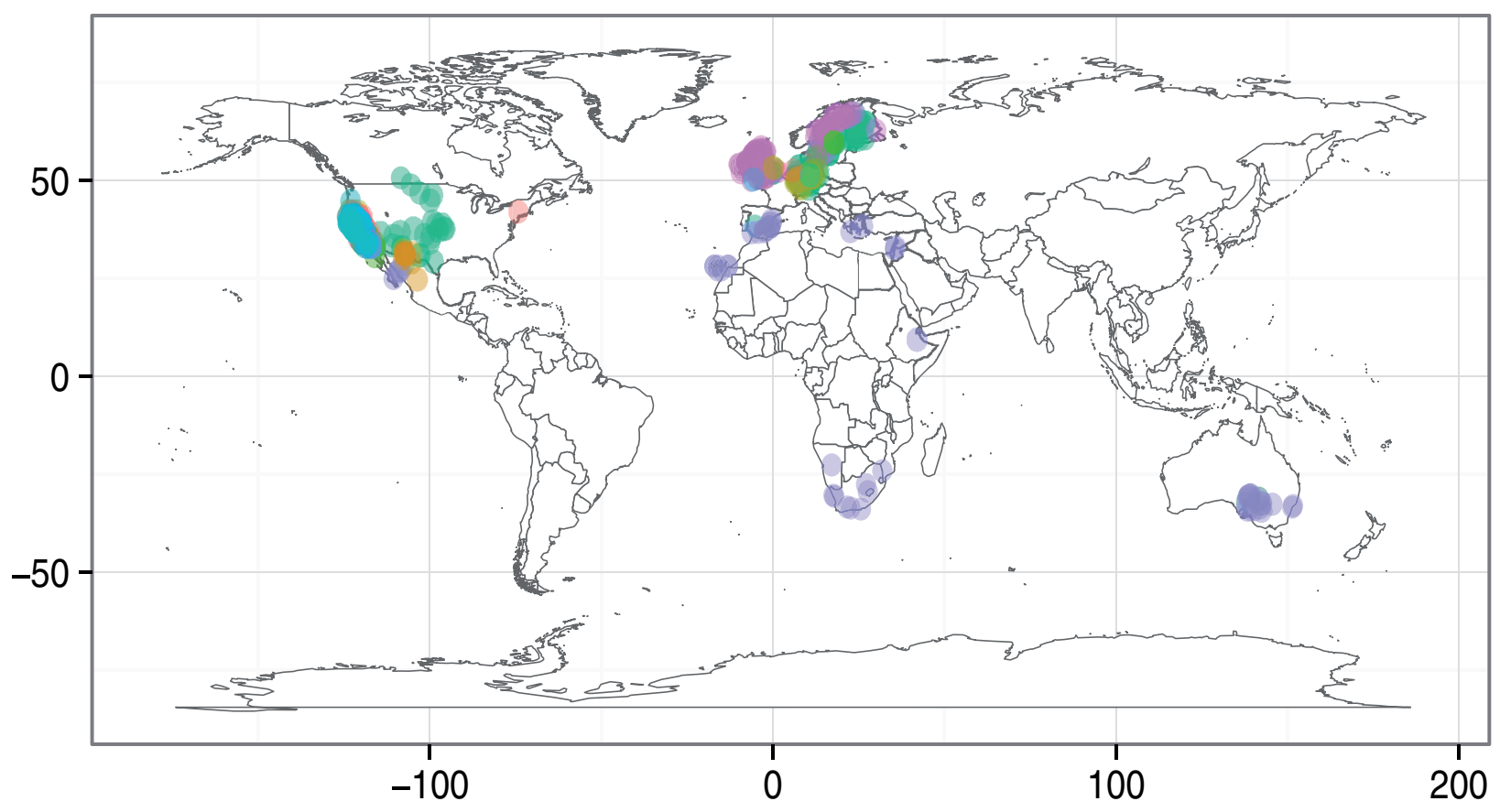
- Abies magnifica
Bartlettia scaposa
Collomia grandiflora
Datura wrightii
- Helianthus annuus
Madia sativa
- Mimulus bicolor
- Nicotiana glauca
- Pinus contorta
- Rosa californica

Figure 2. A map. 


\section{Appendix B. MATCHING SPECIES TABLES WITH DIFFERENT TAXONOMIC RESOLUTION}

Trait-based approaches are a promising tool in ecology. Unlike taxonomy-based methods, traits may not be constrained to biogeographic boundaries ${ }^{27}$ and have potential to disentangle the effects of multiple stressors ${ }^{25}$.

To analyse trait-composition abundance data must be matched with trait databases like ${ }^{26}$. However these two datatables may contain species information on different taxonomic levels and perhaps data must be aggregated to a joint taxonomic level.

taxize can help in this data-cleaning step, providing a reproducible workflow. Here we illustrate this on a small fictitious example.

Suppose we have fuzzy coded trait table with 2 traits with 3 respectively 2 modalities:

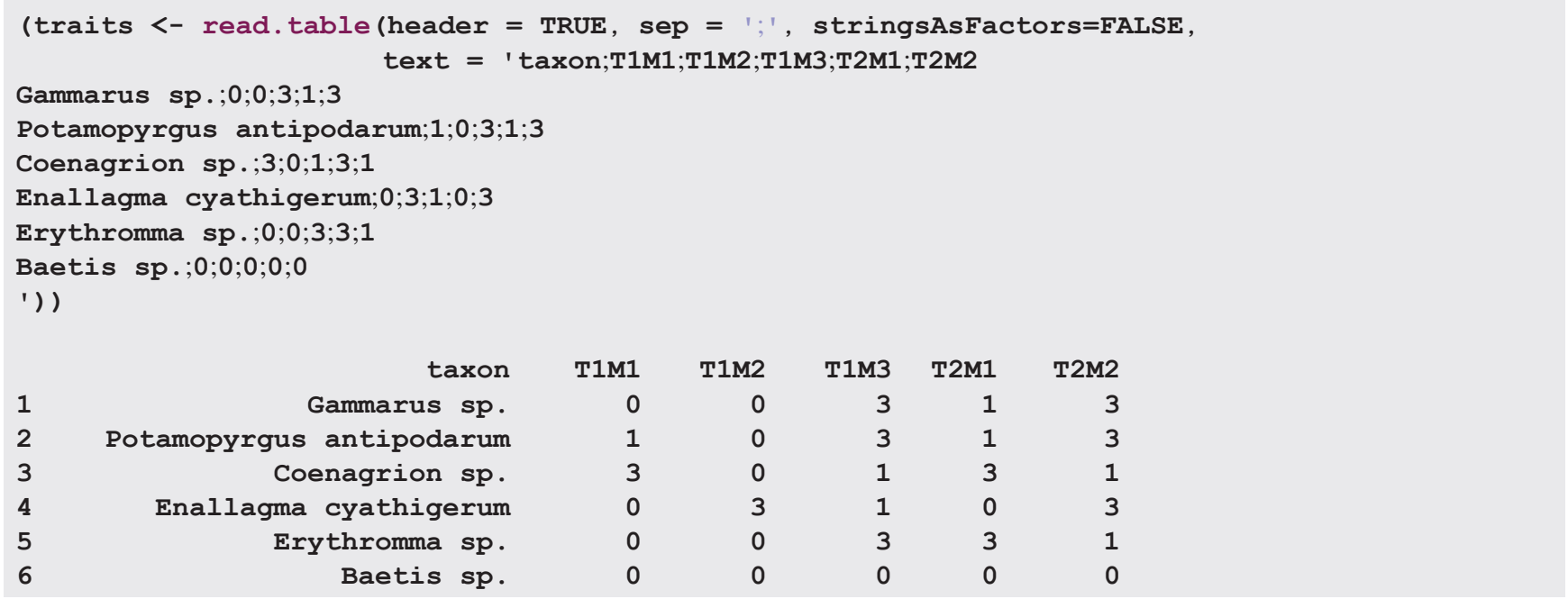

And want to match this to a table with abundances:

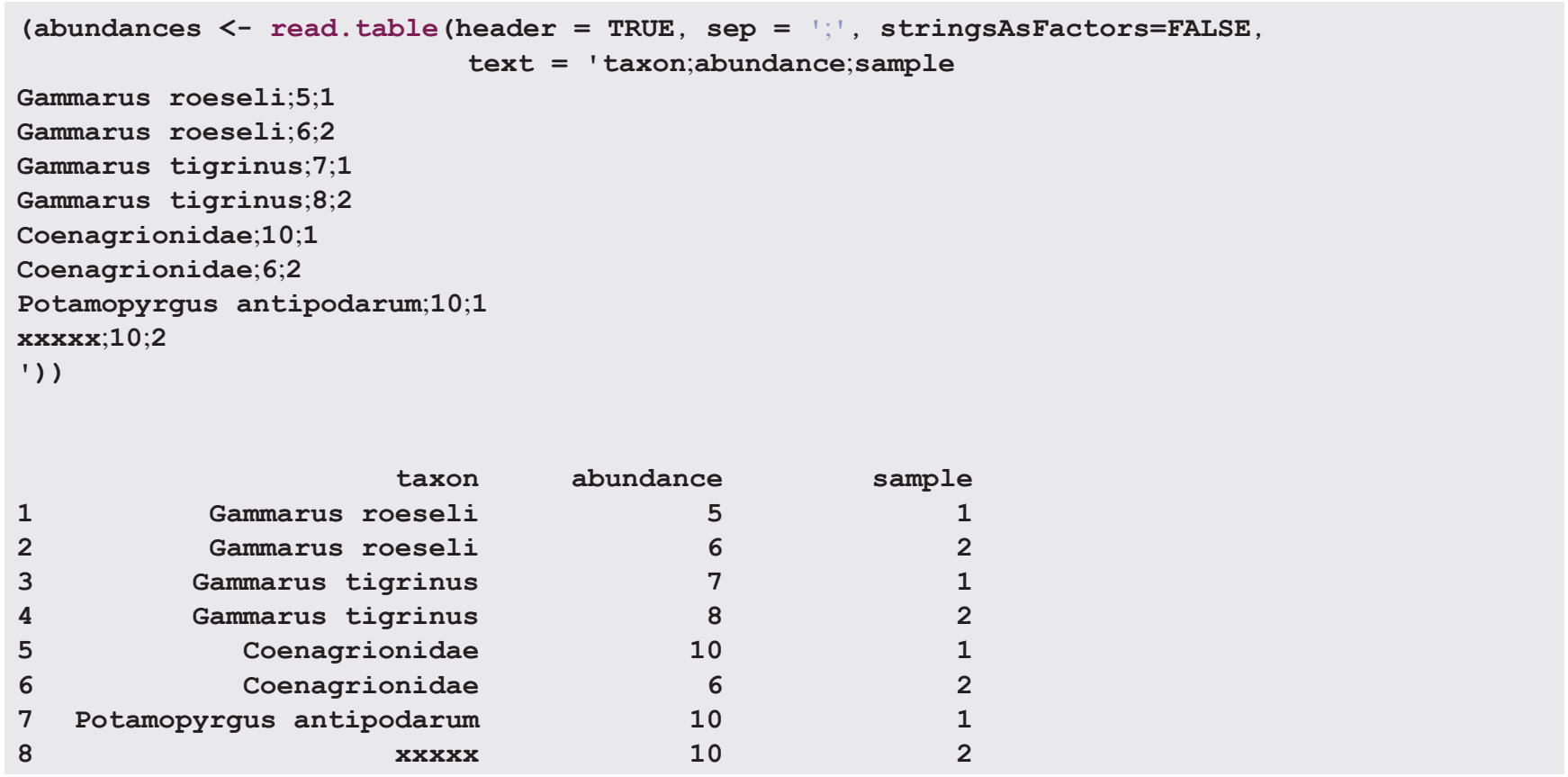

First we do some basic data-cleaning and create a lookup-table, that will link taxa in trait table with the abundance table.

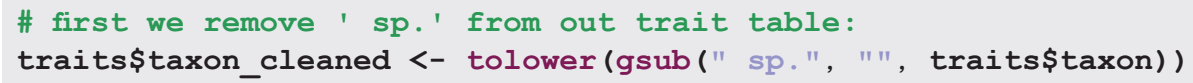


\# since abundance tables can be very long with repeating taxa, we look

\# only at unique taxon names This will be a lookup-table linking taxon

\# names between both tables

lookup <- data.frame (taxon = tolower (unique (abundances\$taxon) $)$, stringsAsFactors = FALSE)

The we query the taxonomic hierarchy for both tables, this will be the backbone of this procedure:

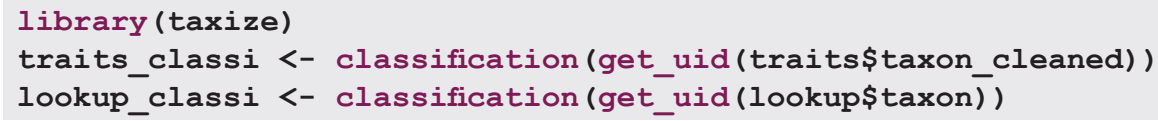

First we look if we can find any direct matches between taxon names:

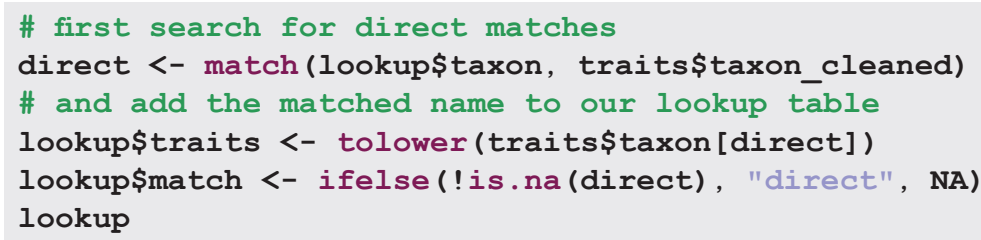

We found a direct match - potamopyrgus antipodarum - so nothing to do here.

Next we look for species which are on a higher taxonomic resolution than our trait table. For these species we will take directly the trait-data since no better information is available.

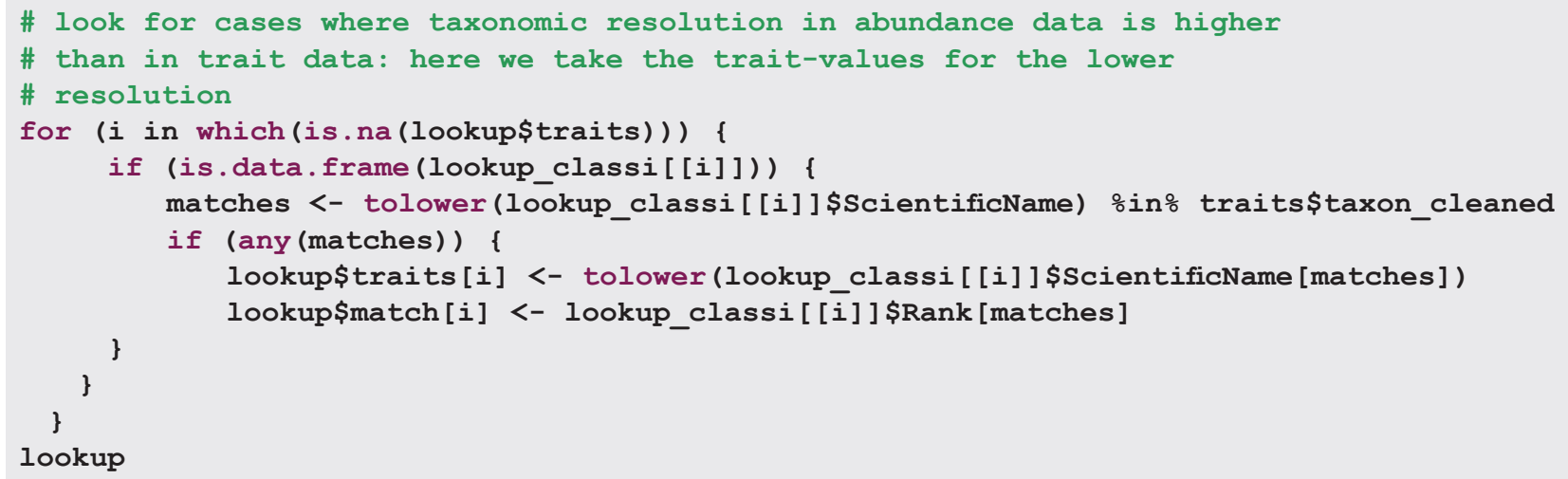

So our abundance data has two Gammarus species, however trait data is only on genus level.

The next step is to search for species were we have to aggregate trait-data, since our abundance data is on a lower taxonomic level. We are walking the taxonomic latter for the species in our trait-data upwards and search for matches with out abundance data. Since we'll have many taxa in the trait-data belonging to one taxon, we'll take the median modality scores as an approximation. Of course also other methods may be used here, e.g. weighting by genetic distance. 


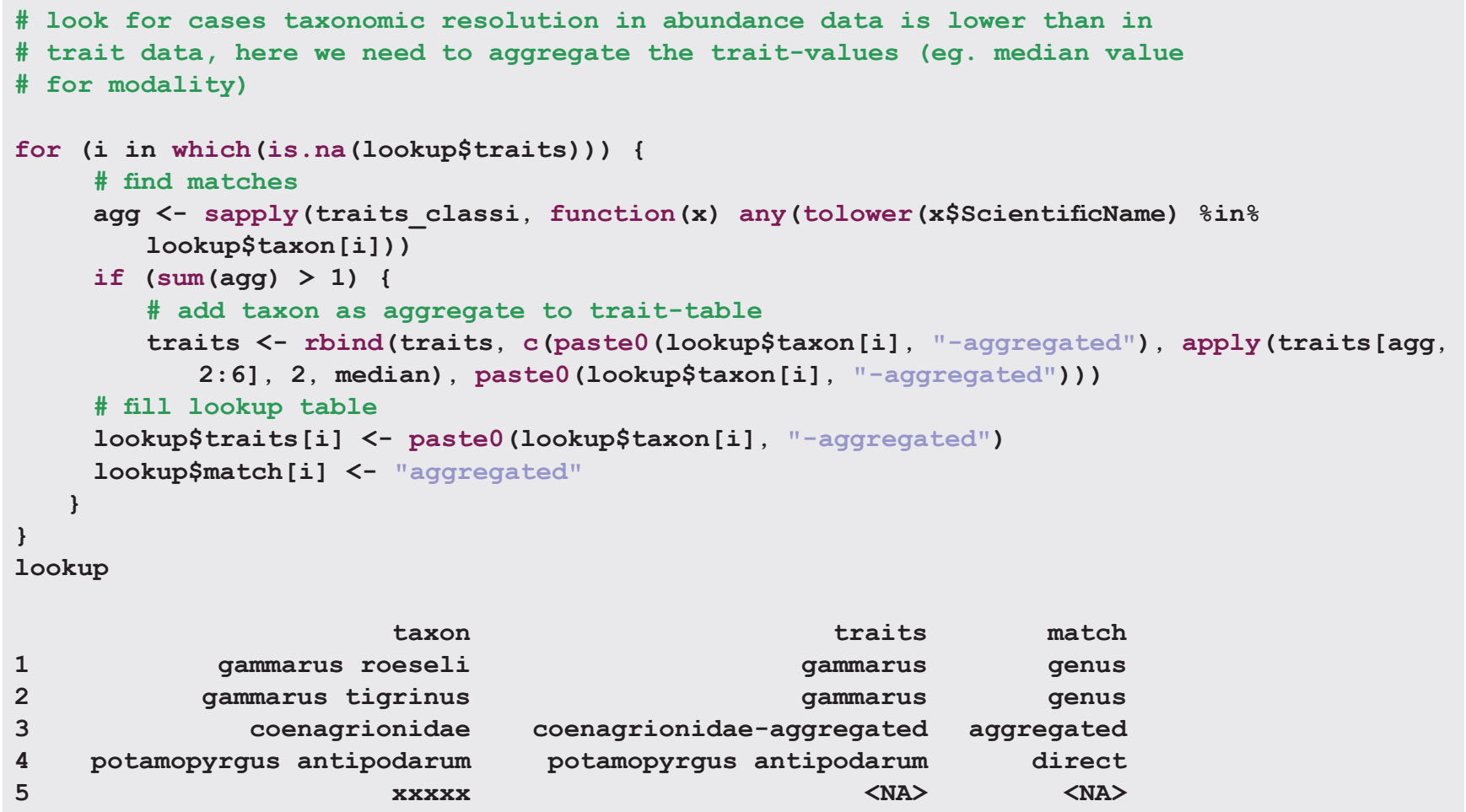

Finally we have only one taxon left - clearly an error. We remove this from our dataset:

abundances <- abundances [ ! abundances $\$$ taxon == lookup\$taxon [is.na (lookup\$traits) ] , ]

Now we can create species $x$ sites and traits $x$ species matrices, which could be plugged into methods to analyse trait responses ${ }^{28}$.

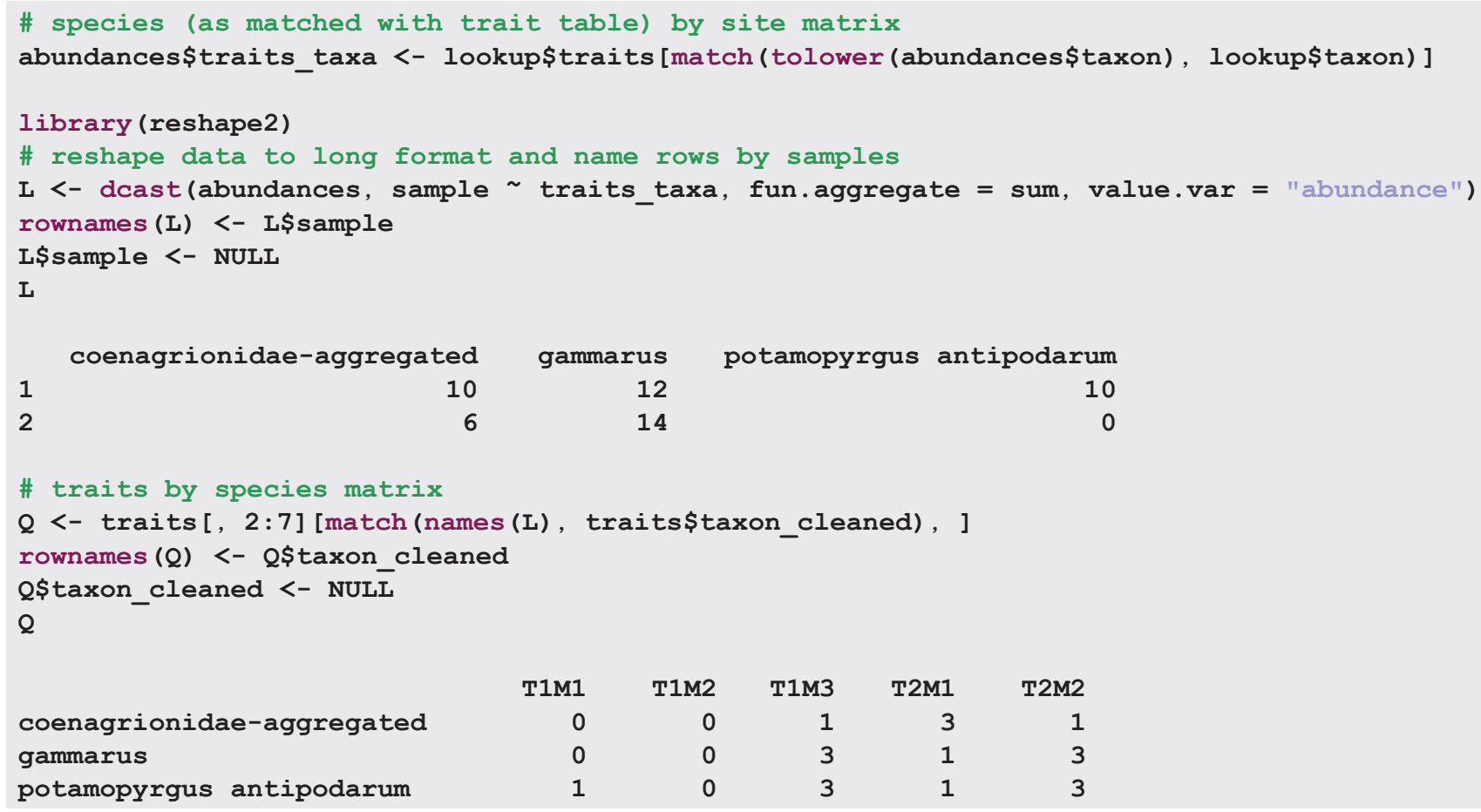

$\begin{array}{rrrrr}\text { T1M1 } & \text { T1M2 } & \text { T1M3 } & \text { T2M1 } & \text { T2M2 } \\ 0 & 0 & 1 & 3 & 1 \\ 0 & 0 & 3 & 1 & 3 \\ 1 & 0 & 3 & 1 & 3\end{array}$




\section{\# check}

all (rownames $(Q)==\operatorname{colnames}(\mathrm{L})$ )

\section{[1] TRUE}

This is just an example how taxonomic APIs (via taxize) could be used to search for matches up- and downwards the taxonomic ladder. We are looking forward to integrate the freshwaterecology.info database www.freshwaterecology.info into taxize, which will facilitate trait-based analyses in R. 


\section{Open Peer Review}

\section{Current Peer Review Status:}

\section{Version 1}

Reviewer Report 24 September 2013

https://doi.org/10.5256/f1000research.2227.r1853

(C) 2013 White E. This is an open access peer review report distributed under the terms of the Creative Commons Attribution License, which permits unrestricted use, distribution, and reproduction in any medium, provided the original work is properly cited.

\section{Ethan White}

Department of Biology, Utah State University, Logan, UT, USA

This software paper describes an R package that provides an integrated $\mathrm{R}$ interface for the APIs of over a dozen taxonomically related web services. This is a valuable contribution because it will save researchers time and energy (for those capable of wrapping the APIs themselves), and will allow scientists who lack the technical knowledge to interact with web services themselves to use this data from R. In addition, some of the functions combine existing APIs in useful ways.

The software was developed using version control, on a public development site (https://github.com/ropensci/taxize_), and using a bug tracker. The code is well modularized and includes an extensive test suite. This level of good software practice is notable for scientific software and is indicative of well built and maintained code. It also has a clearly declared CCO license making it easy for others to use and build on the software.

The software installed easily in R using the standard approach and generally works as expected based on the examples in the paper and on the project's website.

My one major suggestion is to reinforce what the authors' have already suggested in the Conclusions, that it would be an improvement to move to a design that focuses on having a single top-level function for each type of task that the library handles with different data sources being selected using a parameter. This would allow the users to benefit maximally from one of the stronger aspects of this library, which is that it combines access to large numbers of data sources, by making it more of an integrated system and less of a collected set of API wrappers.

\section{Minor issues:}

1. The code snippets are images rather than text. This is probably a limitation of the publishing platform, but it does make it more difficult to learn about the software by executing the code snippets.

2. The following two sentences seem rather tangential to the paper and could be removed: "Science workflows can now easily incorporate text, code, and images in a single executable 
document. Reproducible documents should become mainstream in biology to avoid mistakes, and make collaboration easier."

3. Given that many of the target users of this package will not be particularly familiar with web services and APIs, I would recommend adding another sentence or two to the paragraph on authentication so that readers understand why this is required (i.e., most readers won't understand "users that abuse the API") and what it really is (i.e., an individual login of sorts, similar to a username/password).

4. The section on "Aggregating data to a specific taxonomic rank" refers to an example, but none appears to be present.

Competing Interests: No competing interests were disclosed.

I confirm that I have read this submission and believe that I have an appropriate level of expertise to confirm that it is of an acceptable scientific standard.

Author Response 14 Oct 2013

Scott Chamberlain, Simon Fraser University, Burnaby, Canada

We appreciate Dr. White's comments on our manuscript.

We removed the sentences "Science workflows can now easily incorporate text, code, and images in a single executable document. Reproducible documents should become mainstream in biology to avoid mistakes, and make collaboration easier."

In response to this reviewer's comment about clarification on APIs and authentication, and Dr. Pearse's comments on the same issue, we have added a new appendix (Appendix C) that explains how to use API keys and install the development version of taxize.

This reviewer commented that the section on Aggregating data to a specific taxonomic rank referred to an example, but none appeared to be present. The example is now in the paper.

Competing Interests: No competing interests were disclosed.

Reviewer Report 23 September 2013

https://doi.org/10.5256/f1000research.2227.r1854

(C) 2013 Simpson G. This is an open access peer review report distributed under the terms of the Creative Commons Attribution License, which permits unrestricted use, distribution, and reproduction in any medium, provided the original work is properly cited.

\section{Gavin L. Simpson}

Department of Biology, University of Regina, Regina, SK, Canada 
Chamberlain and Szöcs present the taxize R package, a set of functions that provides interfaces to several web tools and databases, and simplifies the process of checking, updating, correcting and manipulating taxon names for researchers working with ecological/biological data. A key feature that is repeated throughout is the need for reproducibility of science workflows and taxize provides a means to achieve this within the R software ecosystem for taxonomic search.

The manuscript is well-written and nicely presented, with a good balance of descriptive text and discourse and practical illustration of package usage. A number of examples illustrate the scope of the package, something that is fully expanded upon in the two appendices, which are a welcome addition to the paper.

As to the package, I am not overly fond of long function names; the authors should consider dropping the data source abbreviations from the function names in a future update/revision of the package. Likewise there is some inconsistency in the naming conventions used. For example there is the 'tpl_search()' function to search The Plant List, but the equivalent function to search uBio is 'ubio_namebank()'. Whilst this may reflect specific aspects of terminology in use at the respective data stores, it does not help the user gain familiarity with the package by having them remember inconsistent function names.

One advantage of taxize is that it draws together a rich selection of data stores to query. A further suggestion for a future update would be to add generic function names, that apply to a database connection/information object. The latter would describe the resource the user wants to search and any other required information, such as the API key, etc., for example:

$$
\text { foo <- taxizeDB(what = "uBio", key = "1646546164694") }
$$

The user function to search would then be 'search(foo, "Abies")'. Similar generically named functions would provide the primary user-interface, thus promoting a more consistent toolbox at the $\mathrm{R}$ level. This will become increasingly relevant as the scope of taxize increases through the addition of new data stores that the package can access.

In terms of presentation in the paper, I really don't like the way the R code inputs merge with the R outputs. I know the author of Knitr doesn't like the demarcation of output being polluted by the $\mathrm{R}$ prompt, but I do find it difficult parsing the inputs/outputs you show because often there is no space between them and users not familiar with $\mathrm{R}$ will have greater difficulties than I. Consider adding in more conventional indications of R outputs, or physically separate input from output by breaking up the chunks of code to have whitespace between the grey-background chunks. Related, in one location I noticed something amiss with the layout; in the first code block at the top of page 5, the printed output looks wrong here. I would expect the attributes to print on their own line and the data in the attribute to also be on its own separate line.

Note also, the inconsistency in the naming of the output object columns. For example, in the two code chunks shown in column 1 of page 4, the first block has an object printed with column names 'matched_name' and 'data_source_title', whilst camelCase is used in the outputs shown in the second block. As the package is revised and developed, consider this and other aspects of providing a consistent presentation to the user.

I was a little confused about the example in the section Resolve Taxonomic Names on page 4. 
Should the taxon name be "Helianthus annuus" or "Helianthus annus"? In the 'mynames' definition you include 'Helianthus annuus' in the character vector but the output shown suggests that the submitted name was 'Helianthus annus' ( 1 "u") in rows with rownames 9 and 10 in the output shown.

Other than that there were the following minor observations:

1. Abstract: replace "easy" with "simple" in "...fashion that's easy...", and move the details about availability and the URI to the end of the sentence.

2. Page 2, Column 1, Paragraph 2: You have "In addition, there is no one authoritative taxonomic names source...", which is a little clumsy to read. How about "In addition, there is no one authoritative source of taxonomic names..."?

3. Pg 2, C1, P2-3: The abbreviated data sources are presented first (in paragraph 2) and subsequently defined (in para 3). Restructure this so that the abbreviated forms are explained upon first usage.

4. Pg 2, C2, P2: Most R packages are "in development" so I would drop the qualifier and reword the opening sentence of the paragraph.

5. Pg 2, C2, P6: Change "and more can easily be added" to "and more can be easily added" seems to flow better?

6. Pg 5, paragraph above Figure 1: You refer to converting the object to an **ape** *phylo* object and then repeat essentially the same information in the next sentence. Remove the repetition.

7. Pg 6, C1: The header may be better as "Which taxa are children of the taxon of interest".

8. Pg 6: In the section "IUCN status", the term "we" is used to refer to both the authors and the user. This is confusing. Reserve "we" for reference to the authors and use something else ("a user" perhaps) for the other instances. Check this throughout the entire manuscript.

9. Pg 6, C2: in the paragraph immediately below the 'grep()' for "RAG1", two consecutive sentences begin with "However".

10. Pg 7: The first sentence of "Aggregating data...." reads "In biology, one can asks questions...". It should be "one asks" or "one can ask".

11. Pg 7, Conclusions: The first sentence reads "information is increasingly sought out by biologists". I would drop "out" as "sought" is sufficient on its own.

12. Appendices: Should the two figures in the Appendices have a different reference to differentiate them from Figure 1 in the main body of the paper? As it stands, the paper has two Figure $1 \mathrm{~s}$, one on page 5 and a second on page 12 in the Appendix.

13. On Appendix Figure 2: The individual points are a little large. Consider reducing the plotting character size. I appreciate the effect you were going for with the transparency indicating density of observation through overplotting, but the effect is weakened by the size of the individual points.

14. Should the phylogenetic trees have some scale to them? I presume the height of the stems is an indication of phylogenetic distance but the figure is hard to calibrate without an associated scale. A quick look at Paradis (2012) Analysis of Phylogenetics and Evolution with $\mathrm{R}$ would suggest however that a scale is not consistently applied to these trees. I am happy to be guided by the authors as they will be more familiar with the conventions than I.

Competing Interests: No competing interests were disclosed.

I confirm that I have read this submission and believe that I have an appropriate level of 


\section{expertise to confirm that it is of an acceptable scientific standard.}

Author Response 14 Oct 2013

Scott Chamberlain, Simon Fraser University, Burnaby, Canada

We appreciate Dr. Simpson's very thorough comments on our manuscript. The following are responses to Dr. Simpson's comments:

- ...there is some inconsistency in the naming conventions used. For example there is the 'tpl_search()' function to search The Plant List, but the equivalent function to search uBio is 'ubio_namebank()'. Whilst this may reflect specific aspects of terminology in use at the respective data stores, it does not help the user gain familiarity with the package by having them remember inconsistent function names.

We agree that we should definitely improve naming conventions for functions. However, we think it's better to change the function names as needed in an upcoming version of the software after we have had time work on the problem.

- Consider adding in more conventional indications of $R$ outputs, or physically separate input from output by breaking up the chunks of code to have whitespace between the grey-background chunks.

We have used comments (pound signs) for the results of function calls within each code block to indicate output as separate from code input. This way users can copy/paste code directly into R to try it out.

- in one location I noticed something amiss with the layout; in the first code block at the top of page 5, the printed output looks wrong here. I would expect the attributes to print on their own line and the data in the attribute to also be on its own separate line.

This was a problem with the typesetting, and we have fixed it.

- the inconsistency in the naming of the output object columns. For example, in the two code chunks shown in column 1 of page 4, the first block has an object printed with column names 'matched_name' and 'data_source_title', whilst camelCase is used in the outputs shown in the second block.

We agree that we should definitely improve naming conventions for object columns. However, we think it's better to change the column names as needed in an upcoming version of the software after we have had time work on the problem.

- I was a little confused about the example in the section Resolve Taxonomic Names on page 4. Should the taxon name be "Helianthus annuus" or "Helianthus annus"? In the 'mynames' definition you include 'Helianthus annuus' in the character vector but the output shown suggests that the submitted name was 'Helianthus annus' (1 " $u$ ") in rows with rownames 9 and 10 in the output shown. 


\section{Fixed.}

- Abstract: replace "easy" with "simple" in "...fashion that's easy...", and move the details about availability and the URI to the end of the sentence.

\section{Fixed.}

- Page 2, Column 1, Paragraph 2: You have "In addition, there is no one authoritative taxonomic names source...", which is a little clumsy to read. How about "In addition, there is no one authoritative source of taxonomic names..."?

\section{Changed.}

- Pg 2, C1, P2-3: The abbreviated data sources are presented first (in paragraph 2) and subsequently defined (in para 3). Restructure this so that the abbreviated forms are explained upon first usage.

\section{Changed.}

- Pg 2, C2, P2: Most R packages are "in development" so I would drop the qualifier and reword the opening sentence of the paragraph.

\section{Changed.}

- Pg 2, C2, P6: Change "and more can easily be added" to "and more can be easily added" seems to flow better?

\section{Changed.}

- Pg 5, paragraph above Figure 1: You refer to converting the object to an ape phylo object and then repeat essentially the same information in the next sentence. Remove the repetition.

\section{Removed.}

- Pg 6, C1: The header may be better as "Which taxa are children of the taxon of interest".

\section{Changed.}

- Pg 6: In the section "IUCN status", the term "we" is used to refer to both the authors and the user. This is confusing. Reserve "we" for reference to the authors and use something else ("a user" perhaps) for the other instances. Check this throughout the entire manuscript.

\section{Fixed.}

- Pg 6, C2: in the paragraph immediately below the 'grep()' for "RAG1", two consecutive sentences begin with "However". 


\section{Changed.}

- Pg 7: The first sentence of "Aggregating data...." reads "In biology, one can asks questions...". It should be "one asks" or "one can ask"

\section{Changed.}

- Pg 7, Conclusions: The first sentence reads "information is increasingly sought out by biologists". I would drop "out" as "sought" is sufficient on its own.

\section{Changed.}

- Appendices: Should the two figures in the Appendices have a different reference to differentiate them from Figure 1 in the main body of the paper? As it stands, the paper has two Figure 1s, one on page 5 and a second on page 12 in the Appendix.

\section{Fixed.}

- On Appendix Figure 2: The individual points are a little large. Consider reducing the plotting character size. I appreciate the effect you were going for with the transparency indicating density of observation through overplotting, but the effect is weakened by the size of the individual points

Although we agree that the styling of the figure could be improved, we are going to leave it as is because it's not important for understanding the material.

- Should the phylogenetic trees have some scale to them? I presume the height of the stems is an indication of phylogenetic distance but the figure is hard to calibrate without an associated scale. A quick look at Paradis (2012) Analysis of Phylogenetics and Evolution with R would suggest however that a scale is not consistently applied to these trees. I am happy to be guided by the authors as they will be more familiar with the conventions than I.

A scale could be used for sure. However, our focus is on showing readers that they can get data which can be used to make a phylogeny, not on how to properly create and display a phylogeny. Thus, we are leaving the phylogeny as is without a scale.

Competing Interests: No competing interests were disclosed.

Reviewer Report 19 September 2013

\section{https://doi.org/10.5256/f1000research.2227.r1855}

(c) 2013 Pearse $\mathbf{W}$. This is an open access peer review report distributed under the terms of the Creative Commons Attribution License, which permits unrestricted use, distribution, and reproduction in any medium, provided the original work is properly cited. 


\section{Will Pearse}

College of Biological Sciences, University of Minnesota, Minneapolis, MN, USA

The software this article describes is well-written and of use to ecologists. The guide and appendices in this article give a good overview of the features of the package, and are wellwritten. The title and abstract are well-written.

My only serious comment would be that the authors refer to species' taxonomy as if it perfectly reflects species' phylogeny (e.g. the end of the first paragraph in the introduction and the first paragraph in the section "retrieve higher taxonomic names"). More often than not, taxonomy does reflect phylogeny, but a sentence clarifying this distinction somewhere might be helpful.

A few minor things:

Table 1 - "National Center for Biotechnology Information Federhen" - the author's surname, but not the citation, is in the text; "searchbycommonname" and "searchbyscientificname" seem to be the only elements not in alphabetical order in the table.

Phylomatic can now create phylogenies for mammals, not just angiosperms (e.g. "retrieve a phylogeny").

Some sentences could perhaps be linked more neatly in the introduction (e.g. "gives that name as unresolved. But Helianthus", "moved to the cloud. Therefore there is a need", "additional confusion. Last, name"). Similarly, page 3 has some very short paragraphs; could the descriptions of taxonstand and The Plant List be moved to table 1? "Science workflows" or "scientific workflows" (fourth paragraph of introduction)? These are all very minor points!

Perhaps an example of what to type in order to add API keys into the .Rprofile would be helpful, or maybe could just be added to the help file for "ubio_namebank" as this is the function named when it's mentioned.

Competing Interests: No competing interests were disclosed.

\section{I confirm that I have read this submission and believe that I have an appropriate level of expertise to confirm that it is of an acceptable scientific standard.}

Author Response 14 Oct 2013

Scott Chamberlain, Simon Fraser University, Burnaby, Canada

We appreciate Dr. Pearse's comments on our manuscript. We agree that species taxonomy does not equate to phylogenetic history, so we added the following sentence to the first paragraph: "Although taxonomic classifications are human constructs created to understand the real phylogeny of life Icite\{benton2000\}, they are nonetheless essential to organize the vast diversity of organisms." We fixed the citation in Table 1, and reordered the functions in the table so as to be alphabetical. Thanks for pointing out that Phylomatic now accepts mammals in addition to Angiosperm plants - we have adjusted the language accordingly. 
We removed the description of Taxonstand and the Plantlist.org (and associated references) that this reviewer referred to as it wasn't necessary and improves reading.

This reviewer asked for a better explanation of how to use the API keys and the .Rprofile file. In response, we have added a new appendix (Appendix C) that explains using API keys and installing the development version of taxize.

Competing Interests: No competing interests were disclosed.

The benefits of publishing with F1000Research:

- Your article is published within days, with no editorial bias

- You can publish traditional articles, null/negative results, case reports, data notes and more

- The peer review process is transparent and collaborative

- Your article is indexed in PubMed after passing peer review

- Dedicated customer support at every stage

For pre-submission enquiries, contact research@f1000.com 